\title{
$\angle S$ Research Suare \\ Scattering of guided waves propagating through pipe bends based on normal mode expansion
}

\section{Wenjun Wu ( $\nabla$ wuweju@126.com )}

Wuhan University of Technology https://orcid.org/0000-0002-2528-4893

Hao Dong

Wuhan University of Technology

Shangyu Zhang

Wuhan University of Technology

\section{Research Article}

Keywords: Guided wave, Pipe bend, Mode conversion, Normal-mode expansion

Posted Date: March 7th, 2022

DOI: https://doi.org/10.21203/rs.3.rs-1244762/v1

License: @ (i) This work is licensed under a Creative Commons Attribution 4.0 International License. Read

Full License 


\section{Abstract}

The scattering of guided waves propagating through pipe bends is studied by means of normal mode expansion. First, the bi-orthogonality relationship for normal modes in pipe bends is derived, based on which the displacement and stress fields at the interfaces between the straight and curved parts are expanded with the normal modes in both parts. Then, based on the displacement and stress field continuity principle, the scattering problem is regarded as an eigenproblem of a transfer matrix, the solution of which gives the mode conversions at the interfaces. A case study is presented of the low-frequency longitudinal mode incident on a pipe bend, and it is found that the dominant mode conversions are $L(0,1)$ reflection and mode conversion from $L(0,1)$ to $F(1,1)$. The theoretical predictions agree well with results from numerical simulations and experiments.

\section{Introduction}

Because it is highly efficient and can detect zones that would otherwise be inaccessible, guided-wave technology ${ }^{1-3}$ is used extensively for inspecting pipelines. However, practical pipelines always have multiple bends that interfere with the propagation of the incident guided wave and thus complicate the testing signals significantly and even make them impossible to interpret. Therefore, the scattering mechanics of guided waves propagating through pipe bends are essential when inspecting complicated pipelines.

Because of the curved axis of a pipe bend, the wave motion therein is much more complex and must be investigated numerically rather than analytically. Demma et al. ${ }^{4}$ first derived the dispersion curves and mode structures of guided waves in pipe bends with the mode analysis method ${ }^{5}$ in commercial finite-element software, but the dispersion relationship can be calculated only at discrete frequencies. Hayashi et al. ${ }^{6}$ first calculated the dispersion curves of guided waves in pipe bends by using the semi-analytic finite-element method (SAFE), ${ }^{6-10}$ which requires only the pipe cross section to be discretized, thereby turning a threedimensional (3D) problem into a two-dimensional (2D) one and hence saving computational time and memory. A curved cylindrical coordinate system is introduced for the curved pipe region, under which the governing equation of wave motion in pipe bends is derived and then solved with the SAFE method. That method is also applied to dispersion calculations of helical structures ${ }^{8}$ and structures with constant cross sections, such as rails ${ }^{9}$ and square tubes. ${ }^{10}$

Compared with the dispersion curves of guided waves in straight pipes, those for pipe bends exhibit several distinct features, such as cut-off frequencies for the fundamental modes $[L(0,1)$ and $T(0,1)]$, mode splitting, ${ }^{11}$ and mode repulsion. ${ }^{9}$ Demma et al. ${ }^{11}$ studied the mode-splitting feature and gave the explanation that the originally identical modes in straight pipes split into two different modes because of the loss of axisymmetry in pipe bends. Mode repulsion has also been observed in the dispersion curves for curved plates, ${ }^{12,13}$ helical waveguides, ${ }^{8}$ and rails, ${ }^{9}$ among others. Loveday et al. ${ }^{9}$ studied the mode repulsion of guided waves in rails, following which Wu et al. ${ }^{14}$ studied the same in pipe bends. It is found that mode repulsion occurs when the second derivative of frequency with respect to wavenumber approaches infinity as the two curves approach each other. It is also found that mode repulsion occurs only between modes of the same type (e.g., symmetric 
or antisymmetric modes) and not between modes of different types (e.g., symmetric and antisymmetric modes).

Although the propagation characteristics of guided waves in pipe bends are well known, the corresponding scattering mechanics remain less understood. Most studies of scattering mechanics are based on numerical simulations ${ }^{15-19}$ and experiments. ${ }^{20-24}$ By means of 3D finite-element simulation, Aristegui et al. ${ }^{15}$ simulated the $L(0,2)$ mode traveling across pipe bends and observed mode conversions from $L(0,2)$ to $F(1,3)$ and $F(2,3)$. Demma et al. ${ }^{11}$ studied scattering of the torsional $\mathrm{T}(0,1)$ mode and found that it is more likely to be converted to $F(1,2)$. Qi et al. ${ }^{16}$ and Heinlein et al. ${ }^{17}$ investigated reflection of the $T(0,1)$ mode from circumferential and axial defects in pipe bends, respectively. As well as the finite-element method, other numerical methods are also employed: Rudd et al. ${ }^{18}$ used elastodynamic finite integration to simulate guided waves in pipe bends, and Zhou et al. ${ }^{19}$ used the wave finite-element method to study the scattering mechanics of pipe bends.

As for experimental studies, Nishino ${ }^{20}$ used a laser system to generate and sense guided waves in a stainlesssteel pipe, and mode conversions in pipe bends were observed. Also using a laser system, Kim et al. ${ }^{21}$ assessed wall-thinning defects in pipe bends. Verma et al. ${ }^{22}$ generated the $L(0,2)$ mode with magnetostrictive transducers and investigated how the bend angle and radii affected the reflection and transmission coefficients. Similarly, Wu et al. ${ }^{23,24}$ used a magnetostrictive system to study scattering of the $L(0,1)$ and $\mathrm{T}(0,1)$ modes passing through pipe bends.

Based on a bi-orthogonal relationship, the normal-mode expansion (NME) method expresses the wave motion in a waveguide with orthogonal guided wave modes, thereby facilitating force response analysis. Ditri et al. ${ }^{25}$ first derived the bi-orthogonal relationship in hollow cylinders based on the reciprocity theorem, ${ }^{26}$ followed by a generalized mode excitation analysis of pipes with surface traction applied. More specifically, Ditri et al. ${ }^{27}$ analyzed the mode excitation of wedge- and comb-type transducers. Using the same NME method, Zhang et al. ${ }^{28}$ analyzed the force response of elastic hollow cylinders with respect to magnetostrictive loading. Ma et al. ${ }^{29}$ studied the excitation of torsional guided waves in pipes by reversed shear loading. Bakkali et al. ${ }^{30}$ studied the scattering at the junction between straight and curved pipes based on the bi-orthogonal relationship which is simply extended from the bi-orthogonal relationship in plates. Recently, Zhang et al. ${ }^{31}$ used the NME method to study forced guided-wave problems in loading zones and found that the classic NME solution does not satisfy Hooke's law inside the loading zone. To address this shortcoming, Zhang et al. ${ }^{32}$ proposed a modified NME method.

Herein, the NME method is used to study the scattering mechanics of guided waves in pipe bends. In Sec. II, the SAFE modeling of wave motion in pipe bends is introduced briefly, then the bi-orthogonal relationship of normal modes in pipe bends is derived in Sec. III. Based on that relationship, a theoretical study of the scattering mechanics is presented in Sec. IV. To illustrate the theoretical scattering study further, a case study of a low-frequency longitudinal mode incident on a small-radius pipe bend is presented in Sec. V. Finally, in Secs. VI and VII, numerical simulations and experiments, respectively, are reported to validate the theoretical predictions.

\section{li. Safe Modeling Of Wave Motion In Pipe Bends}


As shown in Fig. 1, a quasi-cylindrical coordinate system ${ }^{6}$ is introduced to model the curved hollow cylinder, where the straight $z$ axis in cylindrical coordinates is replaced by a curved $z$ axis along the curvature of the bend. Thus, an arbitrary point $(x, y, z)$ in Cartesian coordinates can be expressed in quasi-cylindrical coordinates $(r, \theta, z)$ as

$$
\left.\begin{array}{c}
x=r \cos \theta \\
y=r \sin \theta \cos \varphi+R(1-\cos \varphi) \\
z=-r \sin \theta \sin \varphi+R \sin \theta \\
R=z^{\prime} / \varphi
\end{array}\right\}
$$

1,

where $R$ is the bending radius.

In quasi-cylindrical coordinates, the stress-displacement relations are rewritten as ${ }^{6}$

$$
\sigma=\mathbf{D}\left[L+L_{r} \frac{\partial}{\partial r}+L_{\theta} \frac{\partial}{\partial \theta}+L_{z^{\prime}} \frac{\partial}{\partial z^{\prime}}\right] \mathbf{u}
$$

2 ,

where $\mathbf{u}=\left[u_{r}, u_{\theta}, u_{z^{\prime}}\right]^{T}$ is the displacement vector, $\sigma=\left[\sigma_{r r^{\prime}}, \sigma_{\theta \theta}, \sigma_{\theta z^{\prime}}, \sigma_{\theta z^{\prime}}, \sigma_{z^{\prime} r^{\prime}}, \sigma_{r \theta}\right]^{T}$ is the stress vector, $\mathbf{D}$ is the constitutive equation,

$L=\left[\begin{array}{ccc}0 & 0 & 0 \\ \frac{1}{r} & 0 & 0 \\ -\frac{\sin \theta}{R h} & -\frac{\cos \theta}{R h} & 0 \\ 0 & 0 & \frac{\cos \theta}{R h} \\ 0 & 0 & \frac{\sin \theta}{R h} \\ 0 & -\frac{1}{r} & 0\end{array}\right], L_{r}=\left[\begin{array}{llll}1 & 0 & 0 \\ 0 & 0 & 0 \\ 0 & 0 & 0 \\ 0 & 0 & 0 \\ 0 & 0 & 1 \\ 0 & 1 & 0\end{array}\right]$, 
$\left.L_{\theta}=\mid \begin{array}{ccc}0 & 0 & 0 \\ 0 & \frac{1}{r} & 0 \\ 0 & 0 & 0 \\ 0 & 0 & \frac{1}{r} \\ 0 & 0 & 0 \\ \frac{1}{r} & 0 & 0\end{array}\right], L_{z^{\prime}}=\left|\begin{array}{ccc}0 & 0 & 0 \\ 0 & 0 & 0 \\ 0 & 0 & \frac{1}{h} \\ 0 & \frac{1}{h} & 0 \\ \frac{1}{h} & 0 & 0 \\ 0 & 0 & 0\end{array}\right|$, and $h=1-\frac{r \sin \theta}{R} .(3)$

Guided waves are assumed to propagate along the curved axis, hence the displacement $\mathbf{u}$ in a pipe bend takes the form

$$
\mathbf{u}=\mathbf{U}(r, \theta) \exp \left(i k z^{\prime}\right) \exp (-i \omega t)
$$

4 ,

where $k$ is the wavenumber, $\omega$ is the angular frequency, and $\mathrm{U}(r, \theta)$ is the interpolated displacement in the cross section of the waveguide. Because the wave motion in the $z$ direction is assumed to be harmonic, finiteelement discretization is required only over the cross section of the pipe bend, with the harmonic wave motion in the $z$ direction included analytically. Because only the cross section is discretized and not a volume, thereby turning a 3D problem into a 2D one, this method decreases the number of nodes significantly and hence saves computational time and memory.

With the rewritten strain-displacement relation [Eq. (2)] and following the standard procedure of the finiteelement method, the governing equation of wave motion in pipe bends can be written as ${ }^{4}$

$$
\left\{\mathbf{K}_{1}+i k \mathbf{K}+k_{3}^{2} \mathbf{K}-\omega^{2} \mathbf{M}\right\} \mathbf{U}=0
$$

5 , where $\mathbf{U}$ is the nodal displacement, $\mathbf{K}_{1}, \mathbf{K}_{2}$, and $\mathbf{K}_{3}$ are the stiffness matrices, and $\mathbf{M}$ is the mass matrix. The stiffness and mass matrices are all real and symmetric, except that $\mathbf{K}_{2}$ is antisymmetric. Let $\mathbf{K}_{2}=i \mathbf{K}$, then $\mathbf{K}_{2}^{\prime}$ is conjugate symmetric. Thus, all the matrices in Eq. (5) can be considered as being conjugate symmetric. The governing equation of Eq. (5) can be regarded as an eigenvalue problem and can be rewritten as

$$
\left(\mathbf{K}_{1}+k \mathbf{K}_{2}^{\prime}+k_{3}^{2} \mathbf{K}\right) \psi=\lambda \mathbf{M} \psi
$$

6 , where $\lambda=\omega^{2}$ is the eigenvalue and $\psi$ is the eigenvector, which also represents the mode structure. Then the wavenumber-frequency dispersion curves and the corresponding mode structure can be computed by solving 
this eigenvalue problem.

\section{lii. Bi-orthogonality Relationship For Normal Modes In Pipe Bends}

The NME method is based on a bi-orthogonal relationship and expresses the wave motion with orthogonal guided wave modes. The bi-orthogonality relationship for normal modes in pipe bends is derived in this section by following the work of Ref. 25 , in which the bi-orthogonality relationship for a straight pipe is deduced.

\section{A. Bi-orthogonality relationship for pipe bends}

The bi-orthogonality relationship for normal modes is derived from the complex reciprocity relation, ${ }^{26}$ which states that

$$
\nabla \cdot\left(\mathbf{v}_{2}^{*} \cdot \mathbf{t}_{1}+\mathbf{v}_{1} \cdot \mathbf{t}_{2}^{*}\right)=0
$$

7, where $\mathbf{V}_{1}, \mathbf{t}_{1}$ and $\mathbf{V}_{2}, \mathbf{t}_{2}$ are the particle velocity and stress fields, respectively, of two different wave motions in a linearly elastic waveguide, and the asterisk represents complex conjugation.

Let $\mathbf{V}_{1}, \mathbf{t}_{1}$ and $\mathbf{V}_{2}, \mathbf{t}_{2}$ be different modes in a pipe bend. In quasi-cylindrical coordinates, they take the forms of

$$
\begin{aligned}
& \mathbf{v}_{1}=\mathbf{v}_{1} e^{-i k_{1} z^{\prime}}, \mathbf{t}_{1}=\mathbf{T}_{1} e^{-i k_{1} z^{\prime}}, \\
& \mathbf{v}_{2}=\mathbf{v}_{2} e^{-i k_{2} z^{\prime}}, \mathbf{t}_{2}=\mathbf{T}_{2} e^{-i k_{2} z^{\prime}},
\end{aligned}
$$

where $k$ is the modal wavenumber, and $\mathbf{V}$ and $\mathbf{T}$ are the particle velocity and stress fields, respectively, over the cross section of the pipe bend. Note that here and hereinafter, the harmonic time dependence $e^{-i w t}$ is omitted for brevity.

Combining Eqs. (7) and (8) gives

$$
\nabla \cdot\left(\mathbf{v}_{2}^{*} \cdot \mathbf{t}_{1}+\mathbf{v}_{1} \cdot \mathbf{t}_{2}^{*}\right)=\nabla \cdot\left\{e^{-i\left(k_{1}-k_{2}^{*}\right) z^{\prime}}\left(\mathbf{V}_{2}^{*} \cdot \mathbf{T}_{1}+\mathbf{V}_{1} \cdot \mathbf{T}_{2}^{*}\right)\right\}=0
$$

9. Integrating Eq. (9) over a slice in the pipe bend (the $\Delta V$ volume in Fig. 1) gives

$$
\iiint_{\Delta V} \nabla \cdot\left\{e^{-i\left(k_{1}-k_{2}^{*}\right) z^{\prime}}\left(\mathbf{V}_{2}^{*} \cdot \mathbf{T}_{1}+\mathbf{V}_{1} \cdot \mathbf{T}_{2}^{*}\right)\right\} d V=0
$$

10.

By using Gauss's divergence theorem, the volume integral in the $\Delta V$ volume becomes the area integral over its surface, i.e., 


$$
\begin{array}{r}
\iiint_{\Delta V} \nabla \cdot\left\{e^{-i\left(k_{1}-k_{2}^{*}\right) z^{\prime}}\left(\mathbf{V}_{2}^{*} \cdot \mathbf{T}_{1}+\mathbf{V}_{1} \cdot \mathbf{T}_{2}^{*}\right)\right\} d V=e^{-i\left(k_{1}-k_{2}^{*}\right) z^{\prime}} \iint_{\partial S_{1}+\partial S_{2}}\left(\mathbf{V}_{2}^{*} \cdot \mathbf{T}_{1}+\mathbf{V}_{1} \cdot \mathbf{T}_{2}^{*}\right) \cdot \hat{n} d s \\
+\left(\begin{array}{l}
\iint_{z S_{1+\Delta z^{\prime}}} e^{-i\left(k_{1}-k_{2}^{*}\right) z^{\prime}}\left(\mathbf{V}_{2}^{*} \cdot \mathbf{T}_{1}+\mathbf{V}_{1} \cdot \mathbf{T}_{2}^{*}\right) \cdot \hat{e}_{z^{\prime}} d s \\
-\iint_{\partial S_{z 1}^{\prime}} e^{-i\left(k_{1}-k_{2}^{*}\right) z^{\prime}}\left(\mathbf{V}_{2}^{*} \cdot \mathbf{T}_{1}+\mathbf{V}_{1} \cdot \mathbf{T}_{2}^{*}\right) \cdot \hat{e}_{z^{\prime}} d s
\end{array}\right)
\end{array}
$$

11 , where \partial $\left\{S_{-} \_1\right\}$ and $\backslash$ partial $\left\{S_{-} 2\right\}$ are the outer and inner surfaces, respectively, of the $\Delta V$ volume, $\backslash$ partial $\left.\left\{S_{-}\left\{z_{\{}{ }_{-} 1\right\}\right\}\right\}$ and $\backslash$ partial $\left\{S_{-}\left\{z\left\{{ }_{-} 1\right\}+\backslash\right.\right.$ Delta $\left.\left.z^{\prime}\right\}\right\}$ are the cross sections of the pipe bend at the $z$ positions of $z\left\{{ }_{-} 1\right\}$ and $z\left\{{ }_{-} 1\right\}+\backslash$ Delta $z^{\prime}$, respectively, $\left\{\backslash\right.$ hat $\left.\{e\}_{-}\left\{z^{\prime}\right\}\right\}$ is the unit vector in the $z$ direction, and $\backslash$ hat $\{n\}$ is the unit normal vector pointing away from the interior volume.

For a free pipe bend, its inner and outer surfaces experience no traction, therefore the first term on the righthand side of Eq. (11) vanishes. Moreover, because the term $\{\backslash \operatorname{mathbf}\{\bigvee\}\}_{-}\{2\}^{\wedge}\{*\} \backslash \operatorname{cdot}\left\{\{\backslash \operatorname{mathbf}\{T\}\}_{-} 1\right\}+$ $\left\{\{\backslash \text { mathbf }\{\bigvee\}\}_{-} 1\right\} \backslash \operatorname{cdot}\{\backslash \operatorname{mathbf}\{T\}\}_{-}\{2\}^{\wedge}\left\{{ }^{*}\right\}$ is independent of $z^{\prime}$, its area integrals in $\backslash$ partial $\left\{S_{-}\left\{z\left\{\left\{_{-} 1\right\}\right\}\right\}\right.$ and Ipartial $\left\{S_{-}\left\{z\left\{z_{-} 1\right\}+\backslash\right.\right.$ Delta $\left.\left.z^{\prime}\right\}\right\}$ are the same. Therefore, Eq. (11) can be written as

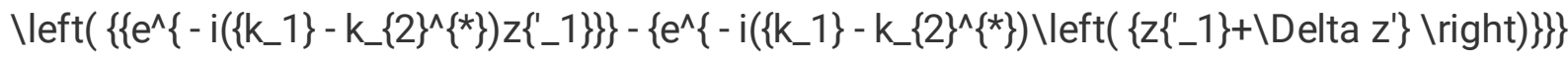

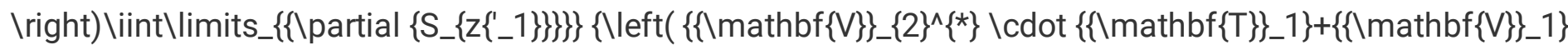
Icdot $\{\backslash$ mathbf $\left.\{T\}\}\{2\}^{\wedge}\{*\}\right\} \backslash$ right $\left.) \backslash \operatorname{cdot}\left\{\{\backslash \text { hat }\{e\}\}_{-}\left\{z^{\prime}\right\}\right\} d s\right\}=0$

12.

Letting \Delta z' \to 0, Eq. (12) still holds and becomes

- i\left } ( \{ \{ k _ { - } 1 \} - k _ { - } \{ 2 \} ^ { \wedge } \{ * \} \} \backslash \operatorname { r i g h t } ) \{ \{ \backslash \operatorname { m a t h b f } \{ P \} \} _ { - } \{ \{ k _ { - } 1 \} , \{ k _ { - } 2 \} \} \} = 0

13 ,

where

$\left\{\{\backslash \text { mathbf }\{P\}\}_{-}\left\{\left\{k_{-} 1\right\},\left\{k \_2\right\}\right\}\right\}\{\backslash \operatorname{text}\{=\}\} \backslash \operatorname{iint}\left\{\{s\}\left\{\backslash \operatorname{left}\left(\left\{\{\backslash \operatorname{mathbf}\{\mathrm{V}\}\}_{-}\{2\}^{\wedge}\{*\} \backslash \operatorname{cdot}\left\{\{\backslash \operatorname{mathbf}\{T\}\}_{-} 1\right\}+\right.\right.\right.\right.$ $\left.\left\{\{\backslash \operatorname{mathbf}\{\mathrm{V}\}\}_{-} 1\right\} \backslash \operatorname{cdot}\{\backslash \operatorname{mathbf}\{T\}\}_{-}\{2\}^{\wedge}\left\{{ }^{\star}\right\}\right\} \backslash$ right $\left.)\right\} \backslash \operatorname{cdot}\left\{\backslash\right.$ hat $\left.\{e\}_{-}\left\{\mathrm{z}^{\prime}\right\}\right\} \mathrm{ds}$

14.

Equation (14) indicates that

$\left\{\{\backslash \operatorname{mathbf}\{P\}\}\left\{\left\{\mathrm{k}_{-} 1\right\},\left\{\mathrm{k}_{\_} 2\right\}\right\}\right\}=\{\backslash \operatorname{text}\{0\}\}$ unless $\left.\mathrm{k}_{-}\{1\}^{\wedge}\{\}\right\}=\mathrm{k}_{-}\{2\}^{\wedge}\left\{{ }^{*}\right\} .(15)$

Equation (15) is the bi-orthogonality relationship for normal modes in pipe bends.

\section{B. Numerical validation}

In this subsection, the bi-orthogonality relationship of Eq. (15) is validated numerically by investigating a stainless-steel pipe with an outer diameter of $22 \mathrm{~mm}$, a thickness of $2 \mathrm{~mm}$, and a bend radius of $50 \mathrm{~mm}$. The material properties of the stainless-steel pipe are given in Table I. 
TABLE I. Material properties of stainless-steel pipe.

\begin{tabular}{|lll|}
\hline Young's modulus & Poisson's ratio & Density \\
\hline $206 \mathrm{GPa}$ & 0.27 & $7930 \mathrm{~kg} / \mathrm{m}^{3}$ \\
\hline
\end{tabular}

The wave motion in the pipe bend is derived using the SAFE method introduced in Sec. II. The cross section of the pipe bend is first discretized with two elements in the radial direction and 48 elements in the circumferential direction. By solving the eigenvalue problem [Eq. (6)], the dispersion relationship for the pipe bend is derived. The group-velocity dispersion curves for the pipe bend are shown in Fig. 2(a), and those for the straight pipe are shown in Fig. 2(b). For comparison, the modes in the pipe bend are denoted as those in the straight pipe but with the addition of the subscript $\mathrm{C}$, as shown in Fig. 2(a). As are clear in Fig. 2(a), the distinct characteristics of the dispersion curves for the pipe bend are (i) the cut-off frequencies evident for the $\mathrm{T}_{\mathrm{C}}(0,1)$ and $\mathrm{L}_{\mathrm{C}}(0,1)$ modes, (ii) the mode-splitting phenomena marked with the frames, and (iii) the moderepulsion phenomena marked with the circles. Note that Fig. 2(a) shows only the positive propagating modes, but all the modes including the negative propagating and non-propagating ones are investigated in the validation of the bi-orthogonality relationship.

The excitation frequency of $30 \mathrm{kHz}$ is chosen to investigate the bi-orthogonality relationship. Also, by solving Eq. (6), the mode structure (eigenvector $\{\backslash$ mathbf $\{\backslash p s i\}\})$ is deduced. Figure 3 shows the displacement distribution along the circumferential direction for the (a) $\left\{\{\backslash \operatorname{text}\{L\}\}_{-}\{\mid \operatorname{text}\{C\}\}\right\}\{\operatorname{text}\{(0,1)\}\},(b)$ $\left.\left\{\{\backslash \operatorname{text}\{T\}\}_{-}\{\mid \operatorname{text}\{C\}\}\right\} \backslash \operatorname{text}\{(0,1)\}\right\}_{,}(\mathrm{c})\left\{\{\mid \operatorname{text}\{F\}\}_{-}\{\mid \operatorname{text}\{C\}\}\right\}\left\{\{\operatorname{text}\{(1,1)\}\}_{-} 1\right\},(\mathrm{d})\left\{\{\backslash \operatorname{text}\{F\}\}_{-}\{\mid \operatorname{text}\{C\}\}\right\}$ $\left.\left\{\{\backslash \operatorname{text}\{(1,1)\}\}_{-} 2\right\},(e)\left\{\{\backslash \operatorname{text}\{F\}\}_{-}\{\mid \operatorname{text}\{C\}\}\right\}\{\backslash \operatorname{text}\{(2,1)\}\}_{-} 1\right\}$, and $\left.(f)\left\{\{\backslash \operatorname{text}\{F\}\}_{-}\{\backslash \operatorname{text}\{C\}\}\right\}\{\backslash \operatorname{text}\{(2,1)\}\}_{-} 2\right\}$ modes.

The term $\left\{\{\backslash \text { mathbf }\{P\}\}_{-}\left\{\left\{\mathrm{K}_{-} 1\right\},\left\{\mathrm{K}_{-} 2\right\}\right\}\right\}$ [Eq. (14)] in the bi-orthogonality relationship is an integral over the cross section, which can be calculated by following the SAFE calculation procedure. For each element on the cross section, we have

\begin } \{ \text { gathered } \} \{ \backslash \text { mathbf } \{ P \} \} _ { - } \{ \{ \{ k _ { - } 1 \} , \{ k _ { - } 2 \} \} \} ^ { \wedge } \{ \operatorname { e } \} \backslash \backslash \operatorname { t e x t } \{ = \} \} \backslash \operatorname { i i n t } \_ \{ \{ \{ \mathrm { s } ^ { \wedge } e \} \} \} \{ \backslash \operatorname { l e f t } ( \{ \{ \backslash \operatorname { m a t h b f } \{ \bigvee \} \} _ { - } \{ 2 \} ^ { \wedge } \{ \{ \mathrm { e } ^ { \star } \} \} \} \backslash \mathrm { cdot } $\left.\left.\left.\{\backslash \operatorname{mathbf}\{T\}\}_{-}\{1\}^{\wedge}\{e\}+\{\backslash \operatorname{mathbf}\{V\}\}_{-}\{1\}^{\wedge}\{e\} \backslash \operatorname{cdot}\{\backslash \operatorname{mathbf}\{T\}\}_{-}\{2\}^{\wedge}\left\{\left\{e^{*}\right\}\right\}\right\} \backslash \operatorname{right}\right)\right\} \backslash \operatorname{cdot}\left\{\{\backslash \text { hat }\{e\}\}_{-}\left\{z^{\prime}\right\}\right\} d\left\{s^{\wedge} e\right\} \backslash$

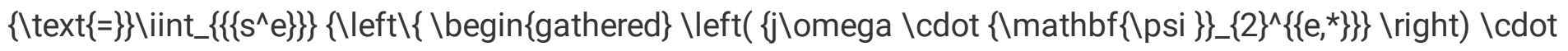
$\backslash$ left $\left\{\{\backslash\right.$ mathbf $\{D\}\} \backslash \operatorname{left}\left(\left\{L+\left\{L_{-} r\right\} \backslash\right.\right.$ frac $\{\backslash$ partial $\}\{\{\backslash$ partial $r\}\}+\left\{L_{-} \backslash\right.$ theta $\} \backslash$ frac $\{\backslash$ partial $\}\{\{\backslash$ partial $\backslash$ theta $\}\}+$ $\left\{L_{-}\left\{z^{\prime}\right\}\right\} \backslash$ frac $\{\backslash$ partial $\}\left\{\left\{\backslash\right.\right.$ partial $\left.\left.\left.z^{\prime}\right\}\right\}\right\} \backslash$ right $\left.)\{\backslash \operatorname{mathbf}\{\backslash p s i\}\}_{-}\{1\}^{\wedge}\{e\}\right\} \backslash$ right $] \backslash h f i l l \backslash \backslash+\backslash$ left $(\{j \backslash$ omega $\backslash$ cdot $\{\backslash$ mathbf $\{\backslash p s i\}\}\left\{\{1\}^{\wedge}\{e\}\right\} \backslash$ right $) \backslash$ cdot $\left\{\backslash\right.$ left $\left[\{\backslash\right.$ mathbf $\{D\}\} \backslash \operatorname{left}\left(\left\{L+\left\{L \_r\right\} \backslash\right.\right.$ frac $\{\backslash$ partial $\}\{\backslash$ partial r $\left.\}\right\}+\left\{L_{-} \backslash\right.$ theta \}$\backslash$ frac $\{\backslash$ partial $\}\{\{\backslash$ partial $\backslash$ theta $\}\}+\left\{L_{-}\left\{z^{\prime}\right\}\right\} \backslash$ frac $\{\backslash$ partial $\}\left\{\left\{\backslash\right.\right.$ partial $\left.\left.\left.z^{\prime}\right\}\right\}\right\} \backslash$ right $)\{\backslash$ mathbf $\left.\{\backslash p s i\}\} \_\{2\}^{\wedge}\{e\}\right\} \backslash$ right $\left.]^{\wedge *}\right\}$ $\backslash$ hfill $\backslash \backslash$ lend $\{$ gathered $\backslash \backslash$ right $\backslash\}\} \backslash$ cdot $\left\{\{\backslash \text { hat }\{e\}\}_{-}\left\{z^{\prime}\right\}\right\} d\left\{s^{\wedge} e\right\} \backslash=$ j\omega $\backslash$ cdot $\backslash$ iint_ $\left\{\left\{\left\{s^{\wedge} e\right\}\right\}\right\}\{\backslash$ left $\backslash\{$

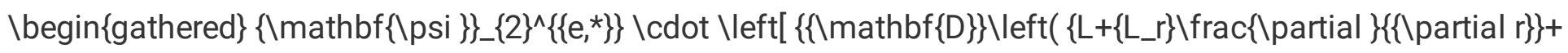

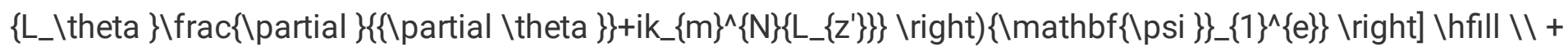
$\{\backslash \text { mathbf }\{\backslash p s i\}\}_{-}\{1\}^{\wedge}\{e\} \backslash \operatorname{codot}\left\{\backslash \operatorname{left}\left[\left\{\{\backslash \operatorname{mathbf}\{D\}\} \backslash \operatorname{left}\left(\left\{L_{+}\left\{L_{-} r\right\} \backslash\right.\right.\right.\right.\right.$ frac $\{\backslash$ partial $\}\{\{\backslash$ partial $r\}\}+\left\{L_{-} \backslash\right.$ theta \}$\backslash$ frac $\{\backslash$ partial $\}\{\{\backslash$ partial $\backslash$ theta $\left.\}\}+i k_{-}\{m\}^{\wedge}\{N\}\left\{L_{-}\left\{z^{\prime}\right\}\right\}\right\} \backslash$ right $)\{\backslash$ mathbf $\{\backslash p s i\}\}\left\{\{2\}^{\wedge}\{e\}\right\} \backslash$ right $\left.]^{\wedge \star}\right\} \backslash$ hfill $\backslash \backslash$ lend\{gathered\} \right } \backslash \} \} \backslash \operatorname { c o t o t } \{ \{ \backslash \text { hat } \{ e \} \} \{ \{ z ^ { \prime } \} \} d \{ s ^ { \wedge } e \} \backslash \backslash \text { end } \{ \text { gathered } \}

16 ,

where the superscript $e$ denotes the element and $\{\backslash \text { mathbf }\{\backslash p s i\}\}_{-}\{\{\}\}^{\wedge}\{e\}$ is the displacement vector of the nodes in that element. The integral in Eq. (16) can be calculated numerically as a Gaussian integral. Then, by 
summing the integrals of all the elements, $\left\{\{\backslash \text { mathbf }\{P\}\}_{-}\left\{\left\{k_{-} 1\right\},\left\{k_{-} 2\right\}\right\}\right\}$ is obtained.

Table II lists the $\left\{\{\backslash \text { mathbf }\{P\}\}_{-}\left\{\left\{k_{-} 1\right\},\left\{k_{-} 2\right\}\right\}\right\}$ values for the normal modes in the pipe bend. These are calculated with the normalized mode structures $\left\{\{\backslash\right.$ mathbf $\{\backslash$ bar $\left.\{\backslash p s i\}\}\} \_k\right\}$, which is defined as follows:

$\left\{\{\backslash\right.$ mathbf $\{\backslash$ bar $\left.\{\backslash p s i\}\}\} \_k\right\}=\backslash f r a c\left\{\left\{\left\{\{\backslash m a t h b f\{\backslash p s i\}\} \_k\right\}\right\}\right\}\left\{\left\{\{\backslash\right.\right.$ mathbf $\left.\left.\left.\{P\}\} \_\{k, k\}\right\}\right\}\right\}$ for propagating modes;

$\left\{\{\backslash\right.$ mathbf $\{\backslash$ bar $\left.\{\backslash p s i\}\}\} \_k\right\}=\backslash$ frac $\left\{\left\{\left\{\{\backslash\right.\right.\right.$ mathbf $\left.\left.\left.\left.\{\backslash p s i\}\} \_k\right\}\right\}\right\}\left\{\left\{\{\backslash \text { mathbf }\{P\}\}_{-}\left\{k,\left\{k^{\wedge \star}\right\}\right\}\right\}\right\}\right\}$ for non-propagating modes. (17)

As given in Table II, the $\left\{\{\backslash\right.$ mathbf $\{P\}\}\left\{\left\{\left\{k_{-} 1\right\},\left\{k_{-} 2\right\}\right\}\right\}$ values for the different modes are zero, thereby validating the bi-orthogonality relationship.

TABLE II. $\left\{\{\backslash\right.$ mathbf $\left.\{P\}\} \_\left\{\left\{k_{-} 1\right\},\left\{k_{-} 2\right\}\right\}\right\}$ values for modes in pipe bend.

\begin{tabular}{|c|c|c|c|c|c|c|}
\hline \multirow{3}{*}{$\left|\mathbf{P}_{k_{1}, k_{2}}\right|$} & \multirow{3}{*}{$\mathrm{L}_{c}(0,1)$} & \multirow{3}{*}{$\mathrm{T}_{c}(0,1)$} & \multirow{3}{*}{$F_{c}(1,1)$} & \multirow{3}{*}{$\mathrm{F}_{\mathrm{c}}(1,1)_{2}$} & Negative & \multirow{2}{*}{$\begin{array}{c}\text { Non-propagating } \\
\text { mode }\end{array}$} \\
\hline & & & & & \multirow{2}{*}{$\mathrm{T}_{c}(0,1)$} & \\
\hline & & & & & & $k=227.59+309.11$ \\
\hline $\mathrm{L}_{c}(0,1)$ & 1 & $1.85 \times 10^{-12}$ & $1.08 \times 10^{-12}$ & $4.39 \times 10^{-12}$ & $4.50 \times 10^{-14}$ & $3.49 \times 10^{-21}$ \\
\hline $\mathrm{T}_{c}(0,1)$ & $1.85 \times 10^{-12}$ & 1 & $3.90 \times 10^{-13}$ & $4.98 \times 10^{-13}$ & $1.11 \times 10^{-13}$ & $129 \times 10^{-12}$ \\
\hline$F_{c}(1,1)_{1}$ & $1.08 \times 10^{-12}$ & $4.39 \times 10^{-12}$ & 1 & $1.51 \times 10^{-11}$ & $2.10 \times 10^{-1.3}$ & $8.14 \times 10^{-22}$ \\
\hline $\mathrm{F}_{c}(1,1)_{2}$ & $439 \times 10^{-12}$ & $4.98 \times 10^{-1.3}$ & $1.51 \times 10^{-11}$ & 1 & $3.42 \times 10^{-1.3}$ & $1.26 \times 10^{-21}$ \\
\hline \multicolumn{7}{|l|}{ Negative } \\
\hline & $4.50 \times 10^{-14}$ & $1.11 \times 10^{-13}$ & $2.10 \times 10^{-1.3}$ & $3.42 \times 10^{-1.3}$ & 1 & $9.03 \times 10^{-23}$ \\
\hline $\mathrm{T}_{c}(0,1)$ & & & & & & \\
\hline
\end{tabular}

Non-propagat

ing

mode $\quad 3.49 \times 10^{-21} \quad 1.29 \times 10^{-12} \quad 8.14 \times 10^{-22} \quad 1.26 \times 10^{-21} \quad 9.03 \times 10^{-23} \quad 1$

$k=227.59+309.11$

\section{Iv. Theoretical Study Of Guided-wave Scattering At Pipe Bends}


Assume that a guided wave excited in the straight part of a pipe propagates through a pipe bend, as shown in Fig. 4. For one pipe bend, there are two interfaces on the propagating path, as marked by $z\left\{{ }^{\prime} \_1\right\}$ and $z\left\{{ }^{\prime} \_2\right\}$ in

Fig. 4. Complicated mode conversions occur at these interfaces, scattering different modes of the guided wave and causing significant confusion with the testing signals.

\section{A. Basic principles for theoretical derivation}

For each interface, the displacement and stress fields over the cross section should be consistent, i.e.,

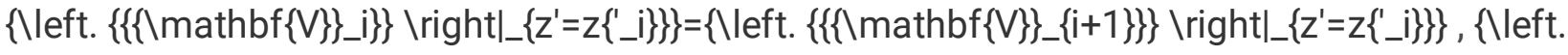
$\left\{\left\{\{\backslash \text { mathbf }\{T\}\}_{-} i\right\}\right\} \backslash$ right $\left.\left.\right|_{-}\left\{z^{\prime}=z\left\{{ }_{-} i\right\}\right\}\right\}=\left\{\backslash\right.$ left. $\left\{\left\{\{\backslash \operatorname{mathbf}\{T\}\} \_\{i+1\}\right\}\right\} \backslash$ right|_\{ $\left.\left.z^{\prime}=z\left\{{ }^{\prime} \_i\right\}\right\}\right\},(i=1,2),(18)$

where the subscript $i$ denotes the ith section of the pipe shown in Fig. 4. According to the NME method, the mode structures at the interfaces can also be expanded with normal modes in either part, i.e.,

$\{\backslash$ left. $\{\backslash$ mathbf $\{\mathrm{V}\}\} \backslash$ right|_\{z'=z\{'_i $\}\}\}=\backslash$ sum $\backslash$ limits_ $\{\mathrm{n}\}\left\{\backslash \operatorname{left}\left(\left\{\left\{\mathrm{a}_{-}\{\mathrm{s}, \mathrm{n}\}\right\}\left\{\{\{\backslash\right.\right.\right.\right.$ mathbf $\{\backslash$ bar $\left.\left.\{\backslash p s i\}\}\}\} \_\left\{\left\{\mathrm{k}_{-}\{\mathrm{s}, \mathrm{n}\}\right\}\right\}\right\}\right\}$ $\backslash$ right $)=\} \backslash$ sum $\backslash$ limits_\{n\} $\left\{\backslash \operatorname{left}\left(\left\{\left\{\mathrm{a}_{-}\{\mathrm{c}, \mathrm{n}\}\right\}\left\{\{\{\backslash \operatorname{mathbf}\{\backslash \operatorname{bar}\{\backslash p s i\}\}\}\}_{-}\left\{\left\{\mathrm{k}_{-}\{\mathrm{c}, \mathrm{n}\}\right\}\right\}\right\}\right\} \backslash\right.\right.$ right $\left.)\right\}$

19,

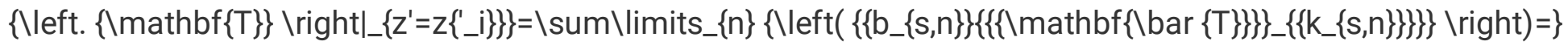
\sum\limits_\{n\} $\left\{\backslash \operatorname{left}\left(\left\{\left\{\mathrm{b} \_\{\mathrm{c}, \mathrm{n}\}\right\}\left\{\{\{\backslash \operatorname{mathbf}\{\backslash \operatorname{bar}\{T\}\}\}\}_{-}\left\{\left\{\mathrm{k}_{-}\{\mathrm{c}, \mathrm{n}\}\right\}\right\}\right\}\right\} \backslash\right.\right.$ right $\left.)\right\}$

20,

where $s$ and $c$ denote the straight pipe and curved pipe, respectively, $a$ and $b$ are the expansion coefficients of the normal modes, and $\{\backslash$ mathbf $\{\backslash$ bar $\{T\}\}\}$ is the normalized stress mode structure, which in the SAFE modeling is defined as

$\{\backslash$ mathbf $\{\backslash$ bar $\{T\}\}\}=\{\backslash$ mathbf $\{D\}\} \backslash$ left $\left[\left\{L+\left\{L_{-} r\right\} \backslash\right.\right.$ frac $\{\backslash$ partial $\}\{\{\backslash$ partial $r\}\}+\left\{L_{-} \backslash\right.$ theta $\} \backslash$ frac $\{\backslash$ partial $\}\{\{\backslash$ partial Itheta $\}\}+\left\{L_{-}\left\{z^{\prime}\right\}\right\} \backslash$ frac $\{\backslash$ partial $\left.\}\left\{\left\{\backslash p a r t i a l z^{\prime}\right\}\right\}\right\} \backslash$ right $]\{\backslash$ mathbf $\{\backslash \operatorname{bar}\{\backslash p s i\}\}\}$

21.

Because the displacement-stress relationship [Eq. (2) or (21)] is nonlinear, the expansion coefficients of the displacement mode structures $\left(a_{n}\right)$ are different from those of the stress mode structures $\left(b_{n}\right)$. However, $b_{n}$ can be calculated according to the displacement-stress relationship.

Based on the bi-orthogonality relationships of both straight pipes ${ }^{[25]}$ and pipe bends, the expansion coefficients are calculated as

$\left\{a_{-}\{s, n\}\right\}=\backslash$ iint_\{s\} $\left\{\backslash\right.$ left $\left(\left\{\left\{\left\{\backslash \text { left. }\left\{\left\{\{\backslash \text { mathbf }\{V\}\}^{\wedge *}\right\}\right\} \backslash \text { right } \mid\right\}_{-}\left\{z^{\prime}=z\left\{{ }^{\prime} \_i\right\}\right\}\right\} \backslash\right.\right.$ cdot $\{\{\{\backslash$ mathbf $\{\backslash$ bar

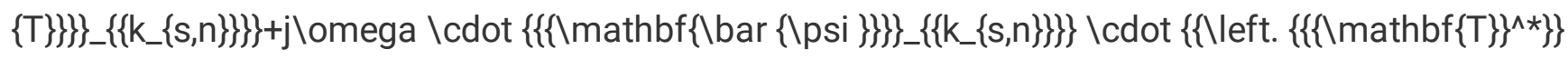
$\backslash$ right $\left.\left.\mid\}_{-}\left\{z^{\prime}=z\left\{{ }^{\prime} i\right\}\right\}\right\}\right\} \backslash$ right $\left.)\right\} \backslash$ cdot $\left\{\backslash\right.$ hat $\left.\{e\}_{-}\left\{z^{\prime}\right\}\right\} d s$

22 ,

$\left\{a_{-}\{c, n\}\right\}=\backslash$ iint_\{s\} $\left\{\backslash\right.$ eft $\left(\left\{\left\{\left\{\backslash \text { left. }\left\{\left\{\{\backslash \text { mathbf }\{V\}\}^{\wedge *}\right\}\right\} \backslash \text { right } \mid\right\}_{-}\left\{z^{\prime}=z\left\{{ }^{\prime} \_i\right\}\right\}\right\} \backslash\right.\right.$ cdot $\{\{\{\backslash$ mathbf $\{\backslash$ bar

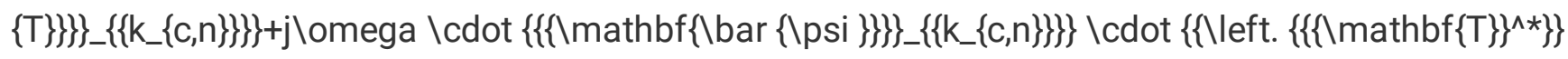
$\backslash$ right $\left.\left.\mid\}_{-}\left\{z^{\prime}=z\left\{{ }^{\prime} i\right\}\right\}\right\}\right\} \backslash$ right $\left.)\right\} \backslash$ cdot $\left\{\backslash\right.$ hat $\left.\{e\}_{-}\left\{z^{\prime}\right\}\right\} d s$

23. 
Note that because the normal modes are essentially the solutions to the governing equation of wave motions in waveguides, the normal modes cannot satisfy two different governing equations for different waveguides simultaneously, indicating that the displacement field of the interface cannot be expressed by modes in straight pipes and pipe bends simultaneously. This is to say, the displacement and stress field continuity principle does not hold in the NME framework. However, the NME method still reveals the inherent connections between the modes in straight pipes and pipe bends, and it gives valuable information about the mode conversions at pipe bends. Therefore, the displacement and stress field continuity principle is assumed to hold in the following derivation.

\section{B. Scattering study for first interface}

At interface $z\left\{{ }^{\prime}-1\right\}$, each mode in straight section 1 can also be expanded with normal modes in curved section 2, i.e.,

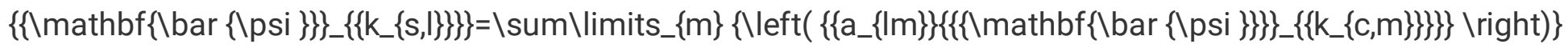
24,

$\left\{a_{-}\{\operatorname{lm}\}\right\}=\backslash \operatorname{iint}\left\{\{s\}\left\{\backslash \operatorname{left}\left(\left\{\left\{\{\{\backslash \operatorname{mathbf}\{\backslash \operatorname{bar}\{\backslash p s i\}\}\}\}^{\wedge *}\right\}_{-}\left\{\left\{\left\{k_{-}\{s, \mid\}\right\}\right\}\right\} \backslash\right.\right.\right.\right.$ cdot $\{\{\{\backslash \operatorname{mathbf}\{\backslash$ bar

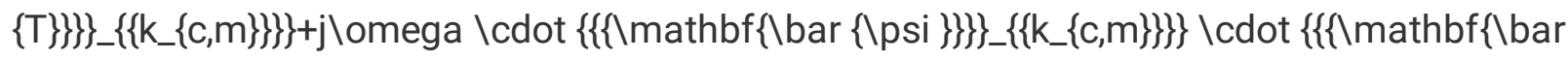
$\left.\left.\{T\}\}\}\}^{\wedge *}\right\}_{-}\left\{\left\{\left\{k_{-}\{s, \mid\}\right\}\right\}\right\}\right\} \backslash$ right $\left.)\right\} \backslash$ cdot $\left\{\backslash\right.$ hat $\left.\{e\}_{-}\left\{z^{\prime}\right\}\right\} d s$

25.

Then, by combining Eqs. (19) and (24) and considering the continuity principle of Eq. (18), we obtain

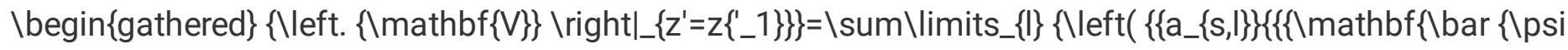

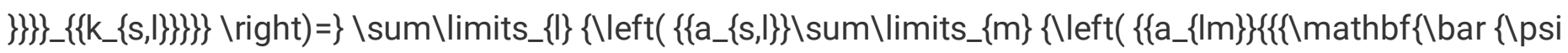

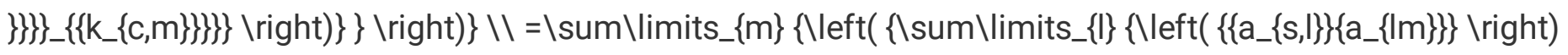
$\left.\left.\left\{\{\{\backslash \text { mathbf }\{\backslash \text { bar }\{\backslash p s i\}\}\}\}_{-}\left\{\left\{k_{-}\{c, m\}\right\}\right\}\right\}\right\}\right\} \backslash$ right $\left.)\right\}=\backslash$ sum $\backslash$ limits_ $\{m\}\left\{\backslash \operatorname{left}\left(\left\{\left\{a_{-}\{c, m\}\right\}\{\{\backslash m a t h b f\{\backslash\right.\right.\right.$ bar $\{\backslash p s i$ \}$\left.\left.\}\}\} \_\left\{\left\{k_{-}\{c, m\}\right\}\right\}\right\}\right\} \backslash$ right $\left.)\right\} \backslash \backslash$ lend $\{$ gathered $\}$

26.

Equation (26) gives the relationship between the expansion coefficients as

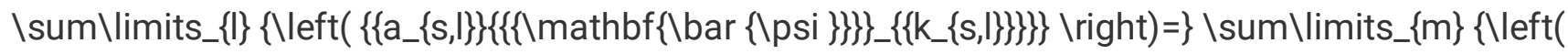

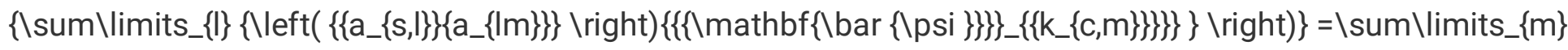
$\left\{\backslash \operatorname{left}\left(\left\{\left\{\mathrm{a}_{-}\{\mathrm{c}, \mathrm{m}\}\right\}\left\{\{\{\mathrm{mathbf}\{\backslash \mathrm{bar}\{\backslash \mathrm{psi}\}\}\}\}_{-}\left\{\left\{\mathrm{k}_{-}\{\mathrm{c}, \mathrm{m}\}\right\}\right\}\right\}\right\} \backslash \operatorname{right}\right)\right\}$

27.,

which can be expressed in matrix form as

$\left\{\{\backslash \text { mathbf }\{A\}\}_{-} m\right\}=\left\{\{\backslash\right.$ mathbf $\left.\left.\{A\}\} \_I\right\}\{\backslash \text { mathbf }\{G\}\}_{-}\{\mid m\}\right\}$

28,

where $\left\{\{\backslash \text { mathbf }\{A\}\}_{-} m\right\}=\backslash \operatorname{left}\left(\left\{a_{-}\{\{c, 1\}\}^{\wedge}\{t\}, a_{-}\{\{c, 2\}\}^{\wedge}\{t\}, \backslash c d o t s\right\} \backslash\right.$ right $),\left\{\{\backslash \text { mathbf }\{A\}\}_{-} \mid\right\}=\backslash$ left ( $\left\{a_{-}\{\{s, 1\}\}^{\wedge}\{i\}, a_{-}\{\{s, 2\}\}^{\wedge}\{i\}, \backslash c d o t s, a_{-}\{\{s, 1\}\}^{\wedge}\{r\}, a_{-}\{\{s, 2\}\}^{\wedge}\{r\}, \backslash c d o t s\right\} \backslash$ right $)$, and $\left\{\{\backslash m a t h b f\{G\}\}_{-}\{\mid m\}\right\}$ is the transfer matrix defined as

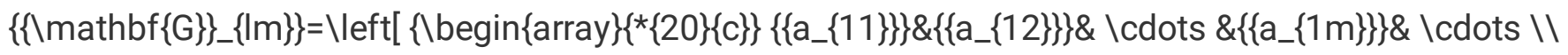

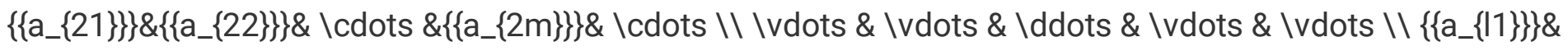




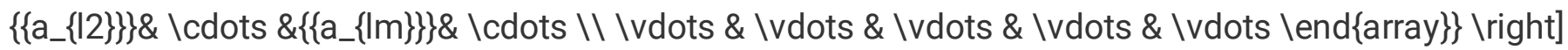
29.

All modes including the incident positive propagating modes, transmitting positive propagating modes, reflecting negative propagating modes, and non-propagating modes should be considered in the calculation. Therefore, the superscripts $i, r$, and $t$ are introduced to denote the incident, reflecting, and transmitting modes, respectively.

Conversely, by expanding each mode in pipe section 2 with normal modes in pipe section 1 and following the same derivation procedure, we have

$\left\{\{\backslash \operatorname{mathbf}\{A\}\}_{-} \mid\right\}=\left\{\{\backslash \operatorname{mathbf}\{A\}\}_{-} m\right\}\left\{\{\backslash \operatorname{mathbf}\{G\}\}_{-}\{\operatorname{Im}\}\right\}=\left\{\{\backslash \operatorname{mathbf}\{A\}\}_{-} \operatorname{m}\right\}\left\{\{\text { mathbf }\{G\}\}_{-}\{\operatorname{lm}\}\right\}^{\prime}$

30 .

Combining Eqs. (29) and (30) gives

$\left\{\{\backslash \operatorname{mathbf}\{A\}\}_{-} \mid\right\}=\left\{\{\backslash \operatorname{mathbf}\{A\}\}_{-} m\right\}\left\{\{\backslash \operatorname{mathbf}\{G\}\}_{-}\{\operatorname{Im}\}\right\}^{\prime}=\left\{\{\backslash \operatorname{mathbf}\{A\}\}_{-} \mid\right\}\left\{\{\backslash \operatorname{mathbf}\{G\}\}_{-}\{\operatorname{lm}\}\right\}\left\{\{\mathrm{mathbf}\{G\}\}_{-}\{\operatorname{lm}\}\right\}^{\prime}$ 31,

which implies that $\left\{\{\backslash \text { mathbf }\{A\}\}_{-} \mid\right\}$is the eigenvector of $\left\{\{\backslash \text { mathbf }\{G\}\}_{-}\{\operatorname{Im}\}\right\}\left\{\{\backslash \text { mathbf }\{G\}\}_{-}\{\operatorname{lm}\}\right\}^{\prime}$ with respect to the eigenvalue of one. Thus, by solving the eigenproblem of $\left\{\{\backslash \operatorname{mathbf}\{G\}\}_{-}\{\operatorname{lm}\}\right\}\left\{\{\backslash \operatorname{mathbf}\{G\}\}_{-}\{\operatorname{Im}\}\right\}^{\prime}$, the expansion coefficients $\left\{\{\backslash\right.$ mathbf $\left.\{A\}\} \_l\right\}$ of guided waves in pipe section 1 can be derived, and $\left\{\{\backslash \mathrm{mathbf}\{\mathrm{A}\}\}_{-} \mathrm{m}\right\}$ can be calculated according to Eq. (28).

In practical inspections, usually a single mode is excited in pipe section 1 . Then, by setting a_ $\{\{s, 1\}\}^{\wedge}\{i\}$ in $\left\{\{\backslash \operatorname{mathbf}\{A\}\}_{-} \mid\right\}$to be one and calculating $\left\{\{\backslash \operatorname{mathbf}\{A\}\}_{-} \mid\right\}$and $\left\{\{\backslash \operatorname{mathbf}\{A\}\}_{-} m\right\}$, the reflecting and transmitting coefficients of guided waves propagating across the first interface are derived.

Considered the acoustic field in either the straight or curved section as being linear, multimode incidence can be treated as multiple single-mode incidences, which can be done by calculating the scattering of each singlemode incidence separately and then linearly superposing these scattering acoustic fields.

\section{Scattering study for second interface}

Because multiple modes are scattered at the first interface, multimode incidence should be considered for the second interface. As mentioned before, multimode incidence is considered as multiple single-mode incidences. For each incident mode $j$, we have

$\left\{\{\backslash \operatorname{mathbf}\{A\}\}_{-}\{n, j\}\right\}=\left\{\{\backslash \operatorname{mathbf}\{A\}\}_{-}\{m, j\}\right\}\left\{\{\backslash \operatorname{mathbf}\{G\}\}_{-}\{m n, j\}\right\}$

32 ,

$\left.\left\{\{\backslash \operatorname{mathbf}\{A\}\}_{-}\{m, j\}\right\}=\left\{\{\backslash \operatorname{mathbf}\{A\}\}_{-}\{m, j\}\right\}\left\{\{\backslash \operatorname{mathbf}\{G\}\}_{-}\{m n, j\}\right\}\{\backslash \operatorname{mathbf}\{G\}\}_{-}\{m n, j\}\right\}^{\prime}$

33 ,

where $\left\{\{\backslash \text { mathbf }\{A\}\}_{-}\{n, j\}\right\}=\backslash \operatorname{left}\left(\left\{a_{-}\{\{s, 1\}\}^{\wedge}\{\{t, j\}\}, a_{-}\{\{s, 2\}\}^{\wedge}\{\{t, j\}\}, \backslash c d o t s\right\} \backslash\right.$ right $)$ are the expansion coefficients of normal modes in straight section 3 , and $\left\{\left\{\backslash \operatorname{mathbf}\left\{\mathrm{A}_{\}}\right\}\right\}_{-}\{\mathrm{m}, j\}\right\}=\backslash \operatorname{left}\left(\left\{\mathrm{a}_{-}\{\mathrm{c}\}^{\wedge}\{\{\mathrm{i}, j\}\}, \mathrm{a}_{-}\{\{\mathrm{c}, 1\}\}^{\wedge}\{\{r, j\}\}, \mathrm{a}_{-}\{\{c, 2\}\}^{\wedge}\{\{r, j\}\}\right.\right.$, $\backslash$ cdots $\} \backslash$ right) are those in curved section 2. Also, by solving the eigenproblem of $\left\{\{\backslash \operatorname{mathbf}\{G\}\} \_\{m n, j\}\right\}$ $\left\{\{\backslash \operatorname{mathbf}\{G\}\}_{-}\{m n, j\}\right\}$, the transmission coefficients $\left\{\{\backslash \text { mathbf }\{A\}\}_{-}\{n, j\}\right\}$ and reflection coefficients $\backslash$ left( 
$\left\{a_{-}\{\{c, 1\}\}^{\wedge}\{\{r, j\}\}, a_{-}\{\{c, 2\}\}^{\wedge}\{\{r, j\}\}, \backslash c d o t s\right\} \backslash$ right $)$ of the jth incident mode scattering at the $z\left\{{ }^{\prime} 2\right\}$ interface are deduced.

By superposing all the scattering acoustic fields, the scattering at the $z\left\{{ }^{\prime}-2\right\}$ interface is obtained. The reflection and transmission coefficients are

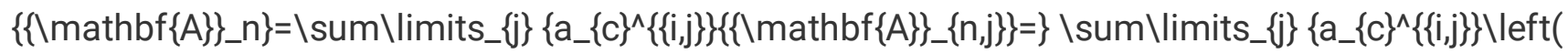
$\left.\left\{a_{-}\{\{s, 1\}\} \wedge\{\{t, j\}\}, a_{-}\{\{s, 2\}\} \wedge\{t, j\}\right\}, \backslash c d o t s\right\} \backslash$ right $\left.)=\right\} \backslash \operatorname{left}\left(\left\{a_{-}\{\{s, 1\}\} \wedge\{t\}, a_{-}\{\{s, 2\}\} \wedge\{t\}, \backslash\right.\right.$ cdots $\} \backslash$ right $)$

34 ,

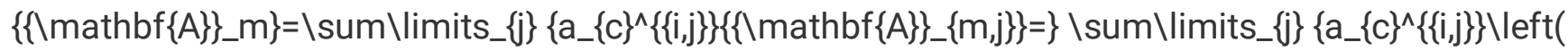
$\left.\left\{1, a_{-}\{\{s, 1\}\}^{\wedge}\{\{t, j\}\}, a_{-}\{\{s, 2\}\} \wedge\{t, j\}\right\}, \backslash c d o t s\right\} \backslash$ right $\left.)=\right\} \backslash \operatorname{left}\left(\left\{a_{-}\{c\}^{\wedge}\{\{i, 1\}\}, a_{-}\{c\}^{\wedge}\{\{i, 2\}\}, \backslash c d o t s a_{-}\{\{s, 1\}\}^{\wedge}\{t\}, a_{-}\{\{s, 2\}\}^{\wedge}\{t\}\right.\right.$, Icdots $\} \backslash$ right)

35 .

The modes reflected at the $z\left\{{ }^{\prime}-2\right\}$ interface then incident negatively on the $z\left\{{ }^{\prime}-1\right\}$ interface, thereby enforcing the reflections of the latter. Because the reflections between the $z\left\{{ }_{-}-1\right\}$ and $z\left\{{ }^{\prime} 2\right\}$ interfaces are rather small in most cases, they are neglected for simplification.

Combining the scattering fields of the $z\left\{{ }_{-} 1\right\}$ and $z\left\{{ }^{\prime}-2\right\}$ interfaces gives the reflection and transmission coefficients $\left(\left\{\{\backslash \text { mathbf }\{A\}\}_{-} \mid\right\}\right.$and $\left.\left\{\{\backslash \text { mathbf }\{A\}\}_{-} n\right\}\right)$ of guided waves traveling through the pipe bend.

\section{Case Study}

In this section, we consider the example of the longitudinal low-frequency axisymmetric $L(0,1)$ mode in a small-bore pipe with a bend. The test pipe is the same as that used in Sec. III B. Assume that the $L(0,1)$ mode with an excitation frequency of $30 \mathrm{kHz}$ is excited in the straight part and then passes through the pipe bend. The mode structures in both the straight pipe and the pipe bend are calculated by using the SAFE method, and the pipe cross section is discretized with two elements in the radial direction and 48 elements in the circumferential direction.

The scattering at the $z\left\{{ }_{-} 1\right\}$ interface is investigated first. The incident $L(0,1)$ mode and all possible reflecting modes are expanded with the normalized modes in the pipe bend according to the bi-orthogonality relationships [Eq. (14)], and then the transfer matrix $\{\{\mid \operatorname{mathbf}\{G\}\}\{\{\mid m\}\}$ is constituted. Theoretically, the nonpropagating reflecting and transmitting modes should be included in the calculation of $\left\{\{\mid \operatorname{mathbf}\{G\}\}_{-}\{\mathrm{m}\}\right\}$. However, because only the propagating modes are of concern in the practical testing scenario, we simplify the calculation of $\left\{\{\backslash \text { mathbf }\{G\}\}_{-}\{\mid \mathrm{m}\}\right\}$ by ignoring the non-propagating modes. Then, we have

$\left\{\{\backslash \text { mathbf }\{G\}\}_{-}\{\operatorname{Im}\}\right\}=\backslash$ left $[\{$ begin $\{$ array $\} *\{20\}\{c\}\}\{-\{\backslash \operatorname{text}\{8225+5691\}\} i\} \&\{\{\backslash \operatorname{text}\{30+262\}\}$ i $\& 0 \& 0 \&\{-$ $\{\backslash \operatorname{text}\{470+2414\}\}\} \backslash \backslash\{\{\backslash \operatorname{text}\{112+920\}\} i\} \&\{\{\backslash \operatorname{text}\{0\}\} \backslash \operatorname{text}\{.87+0\}\} \backslash \operatorname{text}\{.33\}\} ;\} \& 0 \& 0 \&\{-\{\backslash \operatorname{text}\{371\}\}-\{\backslash \operatorname{text}\{$ $298\}\} i\} \backslash\{\{\backslash \operatorname{text}\{50+19\}\} i\} \&\{6+5 i\} \&\{\{\backslash \operatorname{text}\{0\}\}\{\mid \operatorname{text}\{.08+0\}\} \backslash \operatorname{text}\{.09\}\} i\} \&\{15+7 i\} \&\{364-159 i\} \backslash \backslash\{-2.21-$ $0.93 i\} \&\{-0.28+0.23 i\} \&\{1.73+1.92\} \&\{344+149 i\} \&\{-17+7 i\} \backslash \backslash\{-32+20 i\} \&\{-2-24 i\} \&\{-425+42 i\} \&\{47-28 i\} \&\{-$ $1+5 i\} \backslash \backslash\{-539+349 i\} \&\{-35+409 i\} \&\{25-2 i\} \&\{2.79+1.60 i\} \&\{-18+77 i\} \backslash$ end $\{$ array $\}\} \backslash$ right $] \backslash$ times $\left\{10^{\wedge}\{-4\}\right\}$. The input modes, corresponding to the rows of $\left\{\{\backslash \operatorname{mathbf}\{G\}\}_{-}\{\mid \mathrm{m}\}\right\}$, are arranged as the incident $L(0,1)$ and reflecting $L(0,1), F(2,1)_{1}, F(2,1)_{2}, F(1,1)_{1}$, and $F(1,1)_{2}$ modes. The output transmitting modes, corresponding to the columns of $\left\{\{\backslash \text { mathbf }\{G\}\}_{-}\{\mid \mathrm{Im}\}\right\}$, are arranged as the $L_{C}(0,1), F_{C}(2,1)_{1}, F_{C}(2,1)_{2}, F_{C}(1,1)_{1}$, and $F_{C}(1,1)_{2}$ 
modes. $F(1,1)_{1}$ and $F(1,1)_{2}$ are the same modes because they have the same wavenumber. The difference between them is their circumferential orientations of displacement fields, as shown in Fig. 5; this is the same for $F(2,1)_{1}$ and $F(2,1)_{2}$.

Solving the eigenproblem of $\left\{\{\backslash \operatorname{mathbf}\{G\}\}_{-}\{\operatorname{Im}\}\right\}\left\{\{\text { mathbf }\{G\}\}_{-}\{\mid \mathrm{m}\}\right\}$ ' gives the reflection and transmission coefficients:

$\left\{\{\backslash\right.$ mathbf $\left.\{A\}\} \_\mid\right\}=\backslash$ left $(\{\backslash$ begin $\{$ array $\} *\{20\}\{c\}\}\{9936\} \&\{572-718 i\} \&\{-113+1 i\} \& 5 \&\{37+3 i\} \&\{640+48 i\}$ lend\{array\}\} \right) \times $\left\{10^{\wedge}\{-4\}\right\}$,

$\left\{\{\backslash\right.$ mathbf $\left.\{A\}\} \_m\right\}=\backslash \operatorname{left}(\{\backslash$ begin $\{$ array $\} *\{20\}\{c\}\}\{-\{\backslash \operatorname{text}\{8137+5719\}\} i\} \&\{\{\backslash \operatorname{text}\{30+234\}\}$ i\}\&0\&0\& $\{-\{\backslash$ text $\{515$ $+2415\}\}$ i $\backslash$ end $\{$ array $\}\} \backslash$ right $) \backslash$ times $\left\{10^{\wedge}\{-4\}\right\}$.

$\left\{\{\backslash \text { mathbf }\{A\}\}_{-} \mid\right\}$is an eigenvector of $\left\{\{\backslash \operatorname{mathbf}\{G\}\}_{-}\{\operatorname{Im}\}\right\}\left\{\{\text { mathbf }\{G\}\}_{-}\{\operatorname{Im}\}\right\}^{\prime}$ corresponding to the eigenvalue of $0.2892-0.9633 i$, which should be one in theory.

Taking the absolute values of $\left\{\{\backslash\right.$ mathbf $\left.\{A\}\} \_l\right\}$ and $\left\{\{\backslash \text { mathbf }\{A\}\}_{\_} m\right\}$ gives

$\backslash$ left $\mid\left\{\left\{\{\backslash\right.\right.$ mathbf $\left.\left.\{A\}\} \_\mid\right\}\right\} \backslash$ right $\mid=\backslash$ left $(\{\backslash$ begin $\{$ array $\}\{*\{20\}\{c\}\}\{9936\} \&\{918\} \&\{113\} \& 5 \&\{38\} \&\{642\} \backslash$ end array $\}\}$ \right) \times $\left\{10^{\wedge}\{-4\}\right\}$,

$\backslash$ left $\left\{\left\{\{\backslash\right.\right.$ mathbf $\left.\left.\{A\}\} \_m\right\}\right\} \backslash \operatorname{right} \mid=\backslash$ left $(\{\backslash$ begin $\{$ array $\}\{*\{20\}\{c\}\}\{9946\} \&\{\{\backslash$ text $\{236\}\}\} \& 0 \& 0 \&\{\{\backslash$ text $\{2469\}\}\}$ lend\{array\}\} \right) \times $\left\{10^{\wedge}\{-4\}\right\}$.

From the reflection and transmission coefficients of the $z\left\{{ }_{-}-1\right\}$ interface, we conclude the following: (i) 10\% of the incident $L(0,1)$ mode is reflected, while other reflections are rather small; (ii) most of the incident $L(0,1)$ mode is converted into the $L_{C}(0,1)$ mode, part is converted into the $F_{C}(1,1)_{2}$ mode, and all other mode conversions are negligible.

For the $z\left\{{ }^{\prime} 2\right\}$ interface, there are three incident modes. For simplification, the modes with small amplitudes are ignored, and thus only the dominant $L_{C}(0,1)$ and $F_{C}(1,1)_{2}$ modes are considered here. Expanding the $L_{C}(0,1)$ and $F_{C}(1,1)_{2}$ modes with the normalized modes in straight section 3 give the transfer matrices

$$
\begin{aligned}
\mathbf{G}_{\text {mal }} & =\left[\begin{array}{ccccc}
-8225+5691 i & 35-56 i & -1.06+2.56 i & 48+47 i & 191+2599 i \\
789-487 i & 31-43 i & -0.96+2.00 i & -11-12 i & -13-643 i \\
0.77+0.52 i & 7.74+1.51 i & -0.33 & -4.14+9.64 i & -371+176 i \\
0 & -0.06+0.09 i & -1.86+1.80 i & -70+421 i & -5.77-9.25 i \\
0 & -6+15 i & -201+317 i & -4-55 i & 1.00+1.00 i \\
-107+463 i & -70-391 i & 6+16 i & -2.02-0.07 i & -58-54 i
\end{array}\right] \times 10^{-4}, \\
\mathbf{G}_{m a 2} & =\left[\begin{array}{ccccc}
-470+2414 i & -127-595 i & 9+24 i & -247 & -7256-6416 i \\
789-487 i & 31-43 i & -0.96+2.00 i & -11-12 i & -13-643 i \\
0.77+0.52 i & 7.74+1.51 i & -0.33 & -4.14+9.64 i & -371+176 i \\
0 & -0.06+0.09 i & -1.86+1.80 i & -70+421 i & -5.77-9.25 i \\
0 & -6+15 i & -201+317 i & -4-55 i & 1.00+1.00 i \\
-107+463 i & -70-391 i & 6+16 i & -2.02-0.07 i & -58-54 i
\end{array}\right] \times 10^{-4} .
\end{aligned}
$$

The input modes for $L_{C}(0,1)$ are arranged as the incident $L_{C}(0,1)$ and reflecting $L_{C}(0,1), F_{C}(2,1)_{1}, F_{C}(2,1)_{2}$, $F_{C}(1,1)_{1}$, and $F_{C}(1,1)_{2}$ modes. The input modes for $F_{C}(1,1)_{2}$ are arranged as the incident $F_{C}(1,1)_{2}$ and reflecting 
$\mathrm{L}_{C}(0,1), \mathrm{F}_{\mathrm{C}}(2,1)_{1}, \mathrm{~F}_{\mathrm{C}}(2,1)_{2}, \mathrm{~F}_{\mathrm{C}}(1,1)_{1}$, and $\mathrm{F}_{\mathrm{C}}(1,1)_{2}$ modes. The output modes for both cases are arranged as the $L(0,1), F(2,1)_{1}, F(2,1)_{2}, F(1,1)_{1}$, and $F(1,1)_{2}$ modes.

Solving the eigenproblems of $\left\{\{\backslash m a t h b f\{G\}\}_{-}\{m n, 1\}\right\}\left\{\{\backslash m a t h b f\{G\}\}_{-}\{m n, 1\}\right\} '$ and $\left\{\{\backslash m a t h b f\{G\}\}_{-}\{m n, 2\}\right\}$ $\left\{\{\backslash \text { mathbf }\{G\}\}_{-}\{m n, 2\}\right\} '$ gives the reflection and transmission coefficients for the $L_{C}(0,1)$ and $F_{C}(1,1)_{2}$ incidences:

$\left\{\{\backslash \text { mathbf }\{\mathrm{A}\}\}_{-}\{\mathrm{m}, 1\}\right\}=\backslash$ left $(\{\backslash$ begin $\{$ array $\} *\{20\}\{c\}\}\{9948\} \&\{-879+52 \mathrm{i}\} \&\{77-82 \mathrm{i}\} \& 0 \& 0 \&\{400-300 \mathrm{i}\}$ lend\{array\}\} \right) \times $\left\{10^{\wedge}\{-4\}\right\}$, $\{\{\backslash$ mathbf $\{A\}\}\{$ n, 1 $\}\}=\backslash$ left $(\{\backslash$ begin $\{$ array $\}\{*\{20\}\{c\}\}\{-\{\backslash \operatorname{text}\{8240+5730\}\} i\} \&\{\{\backslash \operatorname{text}\{18\}\}-\{\backslash \operatorname{text}\{65\}\} i\} \&\{3 i\} \&$ $\{48+47 i\} \&\{\{\backslash \operatorname{text}\{189+2646\}\} i\} \backslash$ end $\{$ array $\}\} \backslash$ right $) \backslash$ times $\left\{10^{\wedge} 4\right\}$, $\left\{\{\backslash \text { mathbf }\{A\}\}_{-}\{m, 2\}\right\}=\backslash$ left $(\{\backslash$ begin $\{$ array $\} *\{20\}\{c\}\}\{9956\} \&\{722+392 i\} \&\{145-409 i\} \& 0 \& 0 \&\{37-137 i\}$ lend\{array $\}\} \backslash$ right $) \backslash$ times $\left\{10^{\wedge}\{-4\}\right\},\left\{\{\backslash \operatorname{mathbf}\{\mathrm{A}\}\}_{-}\{\mathrm{n}, 2\}\right\}=\backslash \operatorname{left}(\{\backslash$ begin $\{$ array $\}\{*\{20\}\{\mathrm{c}\}\}\{-\{\backslash \operatorname{text}\{398+$ 2399\}\}i\}\&\{ - \{\text $\{118\}\}-\{\backslash \operatorname{text}\{597\}\} i\} \&\{9+24 i\} \&\{-246-1 i\} \&\{-\{\backslash \operatorname{text}\{7198\}\}-\{\backslash$ text $\{6418\}\} i\} \backslash$ end $\{$ array $\}\} \backslash$ right $)$ $\backslash$ times $\left\{10^{\wedge}\{-4\}\right\}$,

which correspond to the eigenvalues of $0.2847-0.9351 i$ and $0.0519+0.9121 i$.

Combining these scattering fields and the transmission coefficients of the $z\left\{{ }^{\prime} \_1\right\}$ interface gives the reflection and transmission coefficients of the $\mathbf{z}\left\{{ }^{\prime} \_2\right\}$ interface:

$\backslash$ begin $\left\{\right.$ gathered $\left\{\{\backslash \operatorname{mathbf}\{\mathrm{A}\}\}_{-} \mathrm{m}\right\}=\backslash \operatorname{left}(\{-\{\backslash \operatorname{text}\{0\}\}\{\backslash \operatorname{text}\{.8137+0\}\}\{\backslash \operatorname{text}\{.5719\}\}$ i $\} \backslash$ right $) \backslash \mathrm{cdot}$ $\left\{\{\backslash \text { mathbf }\{\mathrm{A}\}\}_{-}\{\mathrm{m}, 1\}\right\}+\backslash \operatorname{left}(\{-\{\backslash \operatorname{text}\{0\}\}\{\operatorname{text}\{.0515+0\}\}\{\backslash$ text $\{.2415\}\}$ i $\} \backslash$ right $) \backslash \operatorname{codot}\left\{\{\backslash \operatorname{mathbf}\{\mathrm{A}\}\}_{-}\{\mathrm{m}, 2\}\right\} \backslash \backslash$ $=\backslash$ left $(\{\backslash$ begin $\{$ array $\} *\{20\}\{c\}\}\{-\{\backslash \operatorname{text}\{8095+5689\}\}$ i $\} \&\{-\{\backslash \operatorname{text}\{513+2404\}\}$ i $\&\{\{\backslash \operatorname{text}\{554\}\}-\{\backslash \operatorname{text}\{391\}\}$ i $\} \&$ $\{\{\backslash \operatorname{text}\{76+167\}\}$ i $\} \& 0 \& 0 \&\{-\{\backslash \operatorname{text}\{189+475\}\}$ i $\} \backslash$ end $\{$ array $\}\} \backslash$ right $) \backslash$ times $\left\{10^{\wedge}\{-4\}\right\} \backslash \backslash \backslash$ end $\{$ gathered $\}$, $\backslash$ begin $\{$ gathered $\{\{\backslash$ mathbf $\{A\}\} n\}=\backslash \operatorname{left}(\{-\{\backslash \operatorname{text}\{0\}\}\{\backslash \operatorname{text}\{.8137+0\}\}\{\backslash \operatorname{text}\{.5719\}\} i\} \backslash$ right $) \backslash \mathrm{cdot}$ $\left\{\{\backslash \text { mathbf }\{A\}\}_{-}\{\mathrm{n}, 1\}\right\}+\backslash \operatorname{left}(\{-\{\backslash \operatorname{text}\{0\}\}\{\backslash \operatorname{text}\{.0515+0\}\}\{\backslash \operatorname{text}\{.2415\}\} \mathrm{i}\} \backslash$ right $) \backslash \cot$ t $\left\{\{\backslash \operatorname{mathbf}\{\mathrm{A}\}\}_{-}\{\mathrm{n}, 2\}\right\} \backslash \backslash$ $=\backslash$ left $(\{\backslash$ begin $\{$ array $\} *\{20\}\{c\}\}\{-\{\backslash \operatorname{text}\{2869\}\}-\{\backslash \operatorname{text}\{9595\}\} i\} \&\{\{\backslash \operatorname{text}\{173+65\}\} i\} \&\{-\{\backslash \operatorname{text}\{8\}\}-\{\backslash \operatorname{text}\{2\}\} \mathrm{i}\} \&$

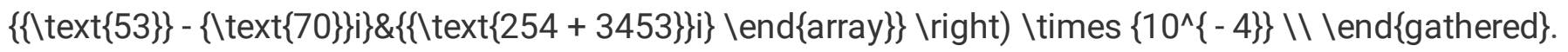

Thus, $\left\{\{\backslash \text { mathbf }\{A\}\}_{-} \mid\right\}$and $\left\{\{\backslash \text { mathbf }\{A\}\}_{-} n\right\}$ give the reflection coefficients (\{\{\mathbf $\left.\left.\left.\{A\}\right\}_{-} r\right\}\right)$ and transmission coefficients (\{\{\mathbf $\left.\left.\{A\}\}_{-} t\right\}\right)$ of the pipe bend at a frequency of $30 \mathrm{kHz}$. Taking the absolute values of $\left\{\{\backslash \text { mathbf }\{A\}\}_{\_} r\right\}$ and $\left\{\{\backslash \text { mathbf }\{A\}\}_{-} t\right\}$ gives

Veft $\left\{\left\{\{\backslash \text { mathbf }\{\text { A }\}\}_{-} r\right\}\right\} \backslash$ right $\mid=\backslash$ left $(\{\backslash$ begin $\{$ array $\}\{*\{20\}\{c\}\}\{0.0918\} \&\{0.0113\} \&\{0.0005\} \&\{0.0038\} \&\{0.0642\}$ \end\{array\}\} \right), }

Vleft $\left\{\left\{\{\backslash\right.\right.$ mathbf $\left.\left.\{A\}\} \_t\right\}\right\} \backslash$ right $\mid=\backslash$ left $(\{\backslash$ begin $\{$ array $\}\{\{20\}\{c\}\}\{1.0014\} \&\{\{\backslash \operatorname{text}\{0\}\}\{\backslash$ text $\{.0185\}\}\} \&\{0.0008\} \&$ $\{0.0088\} \&\{\{\backslash$ text $\{0\}\}\{$ text $\{.3462\}\}\} \backslash$ end\{array\}\} \right),

where the reflection coefficients correspond to reflecting the $L(0,1), F(2,1)_{1}, F(2,1)_{2}, F(1,1)_{1}$, and $F(1,1)_{2}$ modes, and the transmission coefficients correspond to transmitting the $L(0,1), F(2,1)_{1}, F(2,1)_{2}, F(1,1)_{1}$, and $F(1,1)_{2}$ modes.

The reflection and transmission coefficients show that for unit normalized $L(0,1)$ incidence, $10 \%$ of the $L(0,1)$ mode is reflected and more than $100 \%$ of it is transmitted, which means that the law of energy conservation is 
broken here. This happens because the principle of displacement and stress consistency at the interfaces does not hold in the NME framework.

However, although the scattering coefficients are not exact, inherent connections between normal modes in straight pipes and pipe bends are unveiled, and the main mode conversions at pipe bends are predicted correctly. In this case, it can be concluded that most of the incident $L(0,1)$ mode passes through the pipe bend, some of it is reflected, and some converted into the $F_{C}(1,1)_{2}$ mode.

\section{Vi. Numerical Simulations}

To validate the theoretical prediction of $L(0,1)$ mode scattering at pipe bends prior to the experimental validation, numerical simulations were conducted using the commercial finite-element analysis software COMSOL Multiphysics 5.6. The dimensions and material properties of the test pipe were those given in Sec. III B. Figure 6 shows the finite-element modeling of the pipe, which was meshed with two elements in the radial direction and 48 elements in the circumferential direction. The axial mesh spacing was set as $2 \mathrm{~mm}$, which was chosen according to the mesh criterion of more than 20 nodes for the shortest wavelength of interest. The time step was set as $1 \mu$ s according to the criterion of $\backslash$ Delta $t<1 / \operatorname{left}\left(\left\{20\left\{\mathrm{f}_{-}\{\backslash \mathrm{hbox}\{\max \}\}\right\}\right\} \backslash\right.$ right), where $f_{\max }$ is the maximum frequency within a half-power bandwidth.

A five-cycle sinusoidal tone burst modulated with the Hann window function at the excitation frequency of 30 $\mathrm{kHz}$ was applied on the cross section of one end of the pipe in the axial direction. The watchpoints were set on the other end of the pipe, as shown in Fig. 6. Figure 7 shows the time traces of the axial displacement recorded at the watchpoint which locates align to the intrados of the elbow (see Fig. 6): (a) is the complete time trace; (b) is the time trace of axisymmetric modes (the $L(0,1)$ mode in this case) obtained by averaging the displacement of all nodes of the outer surface on the cross section; (c) is the time trace of the $F(1,1)$ mode derived by subtracting the displacement of the watchpoint from that of its symmetrical counterpart.

Figure 7 shows that the recorded signal is mainly decomposed into waveforms of the $L(0,1)$ and $F(0,1)$ modes, indicating that no other remarkable mode conversions occur. Significant $L(0,1)$ bend reflections are observed in Fig. 7(b). The amplitude ratio of the first bend reflection [waveform 1 in Fig. 7(b)] to the first end reflection [waveform 2 in Fig. $7(\mathrm{~b})$ ] is $~ 0.2$. In fact, the first bend reflection is composed of two $L(0,1)$ bend reflections with different propagating routes but with the same flight time: one propagating from the excitation end to the bend, being reflected back to the excitation end, and then propagating through the bend to the receiving end; the other first propagating through the bend to the receiving end, turning back at the end, and then being reflected by the pipe bend. Therefore, $\sim 10 \%$ of the incident $L(0,1)$ mode is reflected by the bend. The converted $F(1,1)$ mode seems rather small compared to the $L(0,1)$ bend reflections, which is contrary to the theoretical prediction that a significant part of the $L(0,1)$ mode is converted into the $F(1,1)$ mode. This is because the $\mathrm{F}(1,1)$ mode has dominant displacements in the radial and circumferential directions but has a much smaller axial displacement (see Fig. 5).

In summary, the numerical simulation results agree well with the theoretical predictions. Although the theoretically derived scattering coefficients are not exact, the dominant mode conversions are obtained correctly. 


\section{Vii. Experimental Validation}

In this section, the scattering of the $L(0,1)$ mode travelling through a bend is studied experimentally. The experimental rig is shown in Fig. 8. The test pipe was the same as that used in Sec. III B; this stainless-steel pipe was bent at its middle by an angle of $90^{\circ}$ by using hot bending. A five-cycle $30-\mathrm{kHz}$ tone burst was generated by an arbitrary function generator (Rigol DG1022) and subsequently magnified by a high-voltage power amplifier (Aigtek ATA-3080). The amplified signal was then sent to the transmitting transducer to excite the longitudinal guided waves in the pipe. The weak guided-wave signals were sensed by the receiving transducer and were pre-amplified and high-pass filtered before being acquired by the data acquisition system (NI PXle-1082).

The transmitting and receiving transducers were placed on the same end of the pipe. Magnetostrictive patch transducers were employed. Four pre-magnetized iron-cobalt alloy strips of $70 \mathrm{~mm}$ length, $5 \mathrm{~mm}$ width, and $0.15 \mathrm{~mm}$ thickness were spaced equally around the circumference and bonded longitudinally on the pipe with epoxy glue. A 40-finger solenoid coil was wound over the patches to transmit and receive the signals.

Figure 9 shows the experimental results. $L(0,1)$ bend reflections are evident in the middle between two successive end reflections, this being because the bend was located at the middle of the pipe. Mode-converted $F(1,1)$ is also observed, which can be confirmed simply by its flight time. The time difference between the $L(0,1)$ end reflection (waveform 1 in Fig. 9) and its successive $F(1,1)$ waveform (waveform 2 in Fig. 9) is $~ 0.33$ $\mathrm{ms}$. For one round trip, the incident $L(0,1)$ travels through the bend twice (forth and back), and hence the mode conversion from $L(0,1)$ to $F(1,1)$ occurs twice. Waveform 2 is the scattered $F(1,1)$ mode when the $L(0,1)$ mode propagates back. According to the dispersion curves (see Fig. 2), the theoretical time difference between waveforms 1 and 2 is $0.3 \mathrm{~ms}$, which agrees well with the experimental result.

Therefore, the experimental result that remarkable reflecting $L(0,1)$ and mode-converted $F(1,1)$ modes are scattered at the bend agrees well with the numerical simulations, thereby validating the theoretical predictions.

\section{Viii. Conclusions}

Herein, the scattering of guided waves propagating across pipe bends was studied. First, the bi-orthogonality relationship of normal modes in pipe bends was derived. Then, based on that relationship and considering that the displacement and stress fields at the interfaces between the straight and curved parts of a pipe should be consistent, the scattering problem was regarded as an eigenproblem of a transfer matrix. By solving this eigenproblem, the mode conversions at the interfaces were deduced. Combining the mode conversions at two interfaces of one bend gave the reflection and transmission coefficients of guided waves traveling through the bend. A case study of a low-frequency longitudinal guided wave (the $L(0,1)$ mode) propagating across a pipe bend was given. Numerical simulations and experiments were further conducted to validate the theoretical predictions.

Because the normal modes are essentially the solutions to the governing equation of wave motions in waveguides, the normal modes cannot satisfy two different governing equations for different waveguides simultaneously, indicating that the principle of consistent displacement and stress fields does not hold in the NME framework. For the case of $L(0,1)$ mode incidence, the theoretical prediction that $\sim 10 \%$ of the incident 
mode is reflected, more than $100 \%$ is transmitted, and a remarkable part is converted into the $F(1,1)$ mode is obviously contrary to the law of energy conservation. However, the NME-based derivation still reveals the inherent connections between normal modes in straight pipes and pipe bends, and it gives valuable information about the mode conversions at pipe bends. It is proved by numerical simulations and experiments that $L(0,1)$ reflection and $L(0,1)-F(1,1)$ conversion are the dominant mode conversions in this case.

\section{Declarations}

\section{Acknowledgments:}

This work is supported by National Natural Science Foundation of China

(Grant Nos. 51709216).

\section{References}

1. J. Rose, Ultrasonic guided waves in solid media (Cambridge University Press, 2014).

2. P. Wilcox, M. Lowe, and P. Cawley, "The effect of dispersion on long-range inspection using ultrasonic guided waves," NDT E. Int. 34, 1-9(2001).

3. J. Lee, J. Achenbach, and Y. Cho, "Use of the reciprocity theorem for a closed form solution of scattering of the lowest axially symmetric torsional wave mode by a defect in a pipe," Ultrasonics 84, 45-52(2018).

4. A. Demma, P. Cawley, and M. Lowe, "Guided waves in curved pipes," Review of Quantitative Nondestructive Evaluation 21, 157-164(2002).

5. P. Wilcox, M. Evans, O. Diligent O, M. Lowe, and P. Cawley, “Dispersion and excitability of guided acoustic waves in isotropic beams with arbitrary cross section". Review of Quantitative Nondestructive Evaluation 21, 203-210(2002).

6. T. Hayashi, K. Kawashima, Z. Sun, and J. Rose, "Guided wave propagation mechanics across a pipe elbow," J. Press. Vesse. T. ASME 127, 322-327(2005).

7. M. Cong, X. Wu, and R. Liu, "Dispersion analysis of guided waves in the finned tube using the semianalytical finite element method," J. Sound. Vib. 401, 114-126(2017).

8. F. Treyssede, "Mode propagation in curved waveguides and scattering by inhomogeneities: Application to the elastodynamics of helical structures," J. Acoust. Soc. Am. 129, 1859-1868(2011).

9. P. Loveday, C. Long, and D. Ramatlo, "Mode repulsion of ultrasonic guided waves in rails," Ultrasonics 84 , 341-349(2018).

10. X. Wan, M. Liu, X. Zhang, H. Fan, P. Tse, M. Dong, X. Wang, H. Wei, C. Xu, and H. Ma, "The use of ultrasonic guided waves for the inspection of square tube structures: Dispersion analysis and numerical and experimental studies," Struct. Health. Monit. 20, 58-73(2020).

11. A. Demma, P. Cawley, and M. Lowe, "Mode conversion of longitudinal and torsional guided modes due to pipe bends," Review of Progress in Quantitative Nondestructive Evaluation CP557, 172-179(2001).

12. X. Bao, P. Raju, and H. Uberall, "Circumferential waves on an immersed, fluid-filled elastic cylindrical shell," J. Acoust. Soc. Am. 105, 2704-2709(1999). 
13. G. Maze, F. Leon, and J. Ripoche, "Repulsion phenomena in the phase-velocity dispersion curves of circumferential waves on elastic cylindrical shells," J. Acoust. Soc. Am. 105, 1695-1701(1999).

14. W. Wu, L. Zhu, and Y. Wang, "Study on mode repulsion of ultrasonic-guided waves in pipe bends," J. Press. Vesse. T. ASME 142, 0314027(2020).

15. C. Aristegui, P. Cawley, and M. Lowe M, "Reflection and mode conversion of guided waves at bends in pipes," Review of Progress in Quantitative Nondestructive Evaluation CP509, 209-216(2000).

16. M Qi, S. Zhou, J. Ni, and Y. Li. “Investigation on ultrasonic guided waves propagation in elbow pipe,” Int. J. Pres. Ves. Pip. 139-140, 250-255(2016).

17. S. Heinlein, P. Cawley, and T. Vogt, "Reflection of torsional $\mathrm{T}(0,1)$ guided waves from defects in pipe bends," NDT E. Int. 93, 57-63(2018).

18. K. Rudd, K. Leonard, J. Bingham, and M. Hinders, "Simulation of guided waves in complex piping geometries using the elastodynamic finite integration technique," J. Acoust. Soc. Am. 121, 14491458(2007).

19. W. Zhou, and M. Ichchou, "Wave propagation in mechanical waveguide with curved members using wave finite element solution," Computer Methods in Applied Mechanics and Engineering 199, 2099-2109(2010).

20. H. Nishino, "A feasibility study on pipe inspection using ultrasonic guided waves for maintenance of nuclear power plants," International Symposium on the Ageing Management \& Maintenance of Nuclear Power, 184-198(2010).

21. D. Kim, Y. Cho, and J. Lee, "Assessment of wall-thinning in carbon steel pipe by using laser-generated guided wave," Nuclear Eng. Tech. 42, 546-551(2010).

22. B. Verma, T. Mishra, K. Balasubramaniam, and P. Rajagopal, "Interaction of low-frequency axisymmetric ulstraonic guided waves with bends in pipes of arbitrary bend angle and general bend radius," Ultrasonics 54, 801-808(2014).

23. W. Wu, Y. Wang, L. Chen, and H. Geng, "The effect of pipe bend on low-frequency longitudinal mode guided wave propagation," Acta Acustica 42, 95-102(2017)(in Chinese).

24. W. Wu, and J. Wang, "Effect of pipe bends on the low-frequency torsional guided wave propagation," Archives of Acoustics 45, 385-391(2020).

25. J. Ditri, and J. Rose, "Excitation of guided elastic wave modes in hollow cylinders by applied surface tractions," J. Appl. Phys. 72, 2589-2597(1992).

26. B. Auld, Acoustic Fields and Waves in Solids (John Wiley and Sons Press, New York, 1973).

27. J. Ditri, and J. Rose, "Generation of guided waves in hollow cylinders by wedge and come type transducers," Review of Progress in Quantitative Nondestructive Evaluation 12, 211-218(1993).

28. X. Zhang, Z. Tang, F. Lv, and X. Pan, "Excitation of axisymmetric and non-axisymmetric guided waves in elastic hollow cylinders by magnetostrictive transducers," Journal of Zhejiang University-SCIENCE A (Applied Physics \& Engineering) 17, 215-229(2016).

29. S. Ma, Y. Wang, K. Liu, and Z. Wu, "Analysis of torsional guided waves excitation in pipes by co-rotating and reversed shear loading," Acta Acustica 39, 68-74(2014)(in Chinese).

30. M. Bakkali, A. Lhémery, V. Baronian, and S. Grondel, "A modal formulation for the propagation of guided waves in straight and curved pipes and the scattering at their junction," Journal of Physics: Conference 
Series 498, 012010(2014).

31. P. Zhang, and J. Qu, "Forced guided waves in linearly Eelastic plates (I) - An examination of the normalmode expansion method," Ultrasonics 108, 106231(2020).

32. P. Zhang, and J. Qu, “Forced guided waves in linearly elastic plates (II) - A modified normal-mode expansion method," Ultrasonics 108, 106232(2020).

\section{Figures}

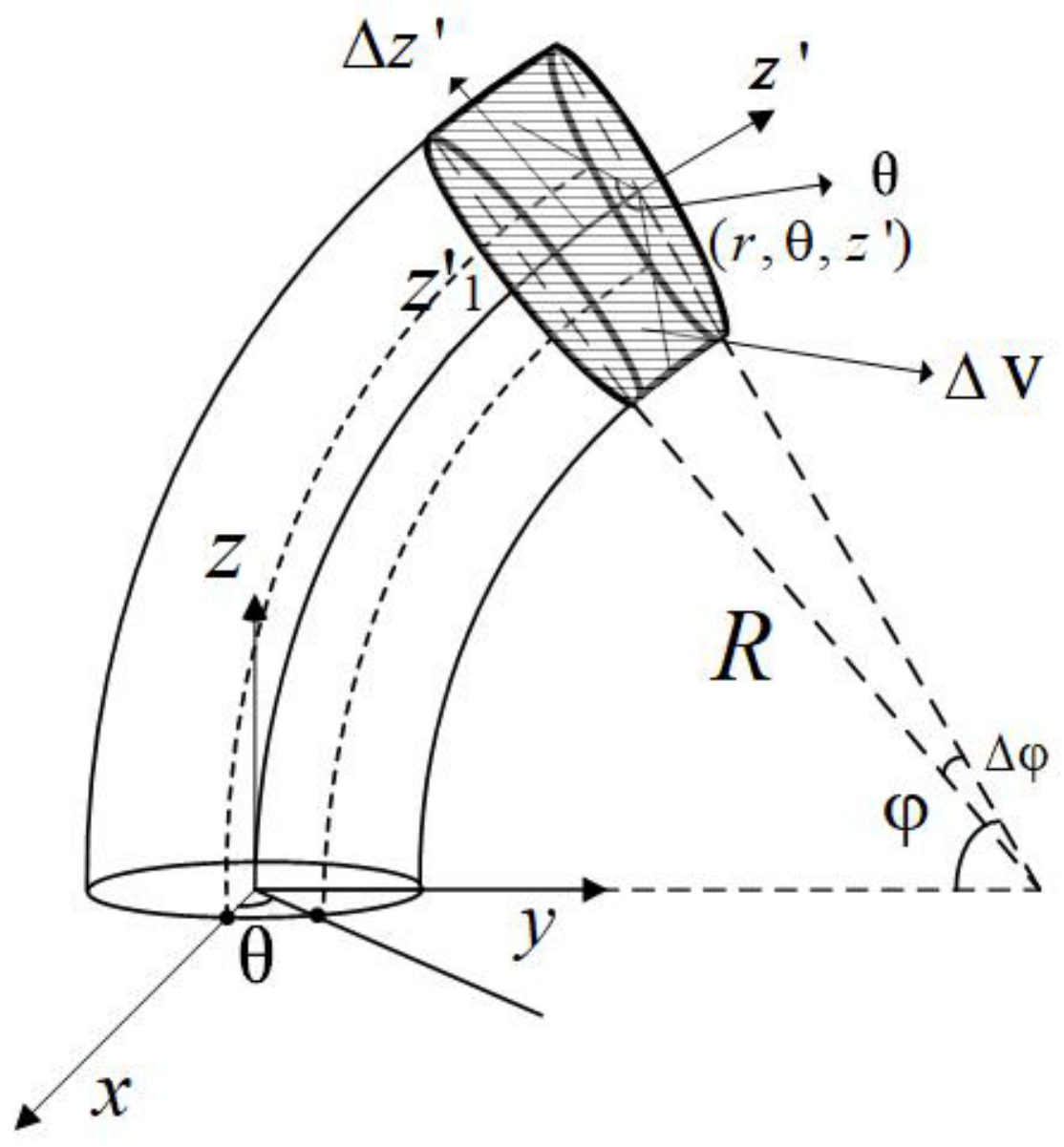

Figure 1

Quasi-cylindrical coordinate system. ${ }^{6}$ 


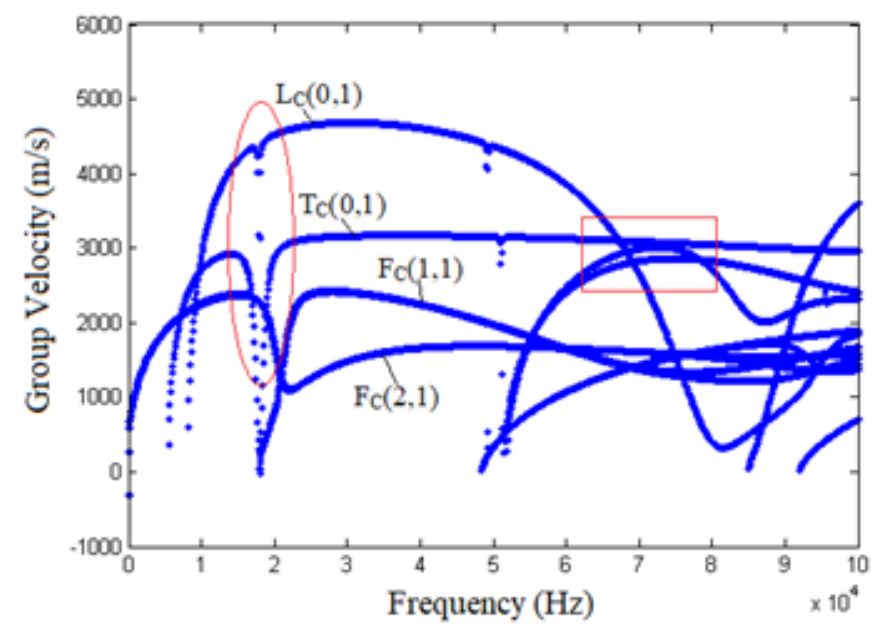

(a) Pipe bend

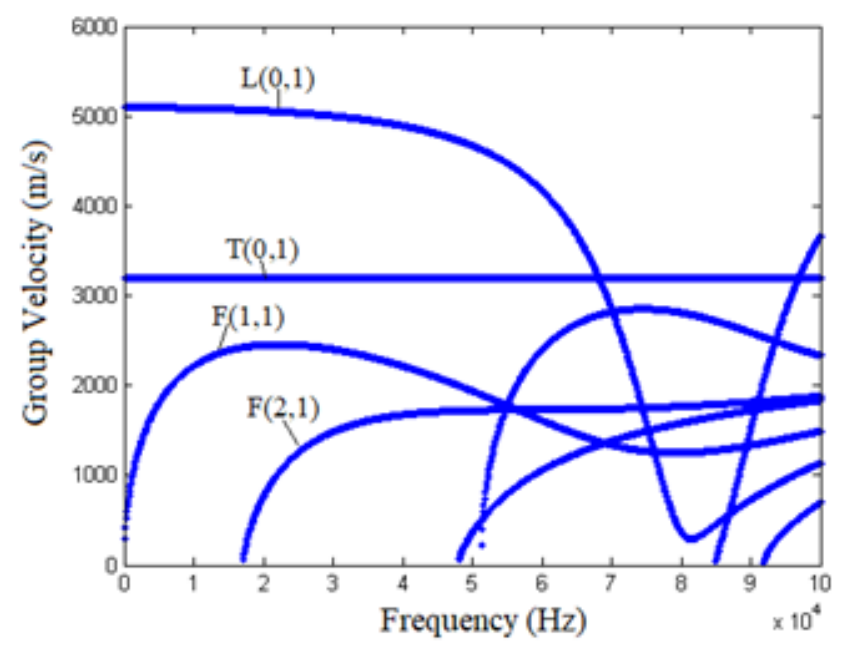

(b) Straight pipe

Figure 2

Group-velocity dispersion curves for (a) a pipe bend and (b) a straight pipe. 

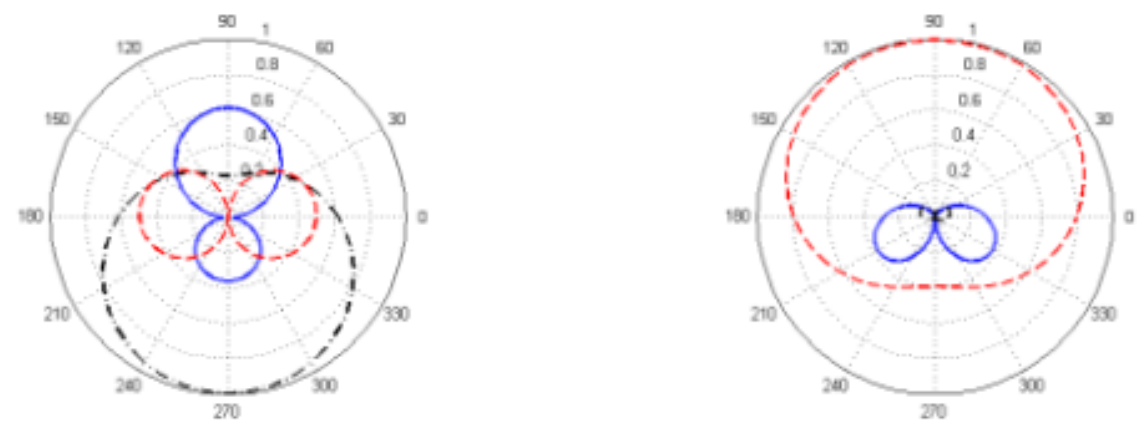

(a) $\quad \mathrm{L}_{\mathrm{c}}(0,1)$

(b) $\quad \mathrm{T}_{\mathrm{c}}(0,1)$
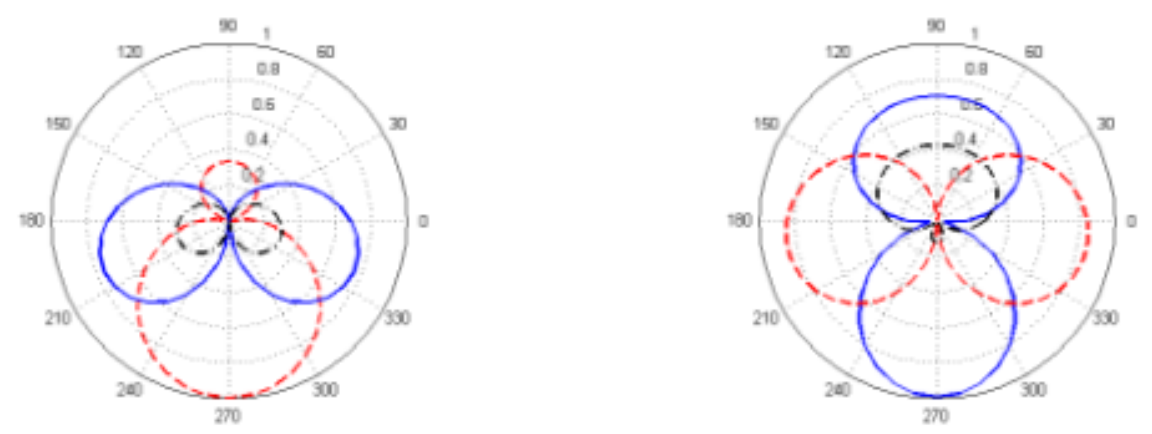

(c) $\quad \mathrm{F}_{\mathrm{c}}(1,1)_{1}$

(d) $\quad \mathrm{F}_{\mathrm{C}}(1,1)_{2}$
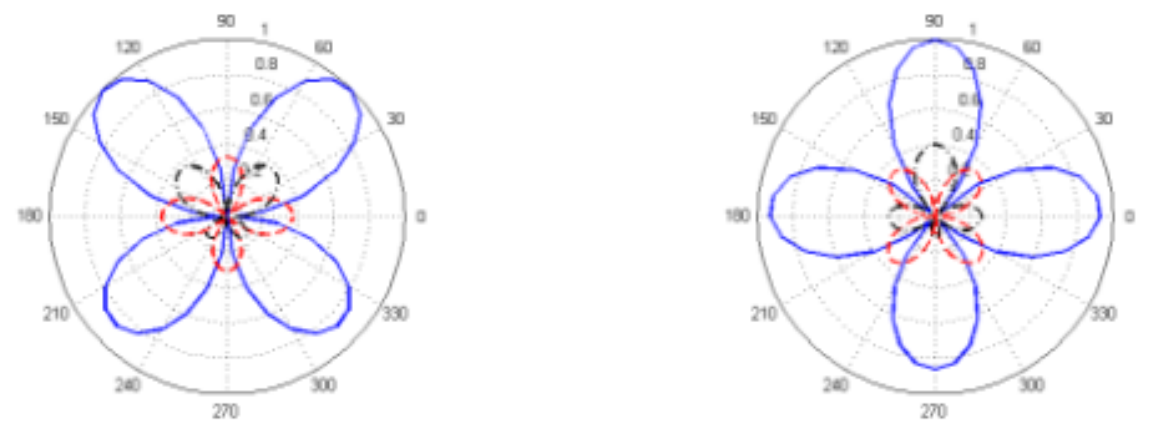

(e) $\quad F_{c}(2,1)$

(f) $\quad \mathrm{F}_{\mathrm{C}}(2,1)_{2}$

\section{Figure 3}

Displacement distributions along circumferential direction (the blue solid, red dotted, and black dashed-dotted lines show the displacements in the radial, circumferential, and axial directions, respectively). 


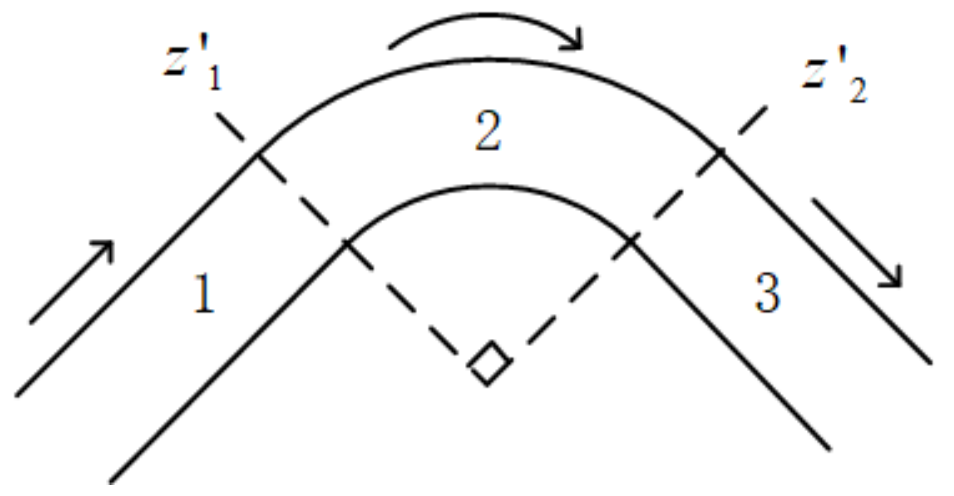

Figure 4

Schematic of guided wave traveling through a bend.

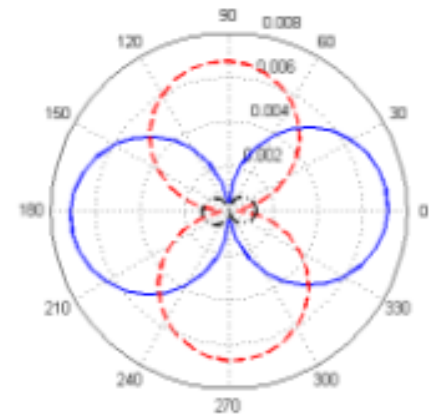

(a) $\quad \mathrm{F}(1,1)_{1}$

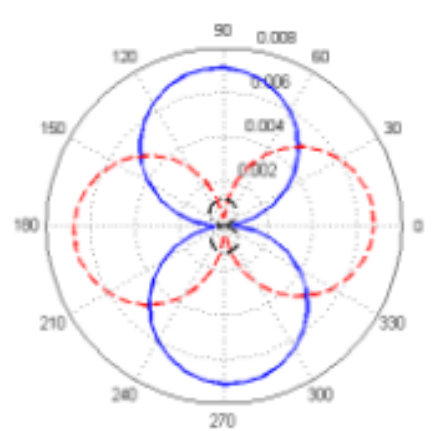

(b) $\quad \mathrm{F}(1,1)_{2}$

\section{Figure 5}

Displacement distributions of (a) $F(1,1)_{1}$ and (b) $F(1,1)_{2}$ along circumferential direction (the blue solid, red dotted, and black dashed-dotted lines show the displacements in the radial, circumferential, and axial directions, respectively). 


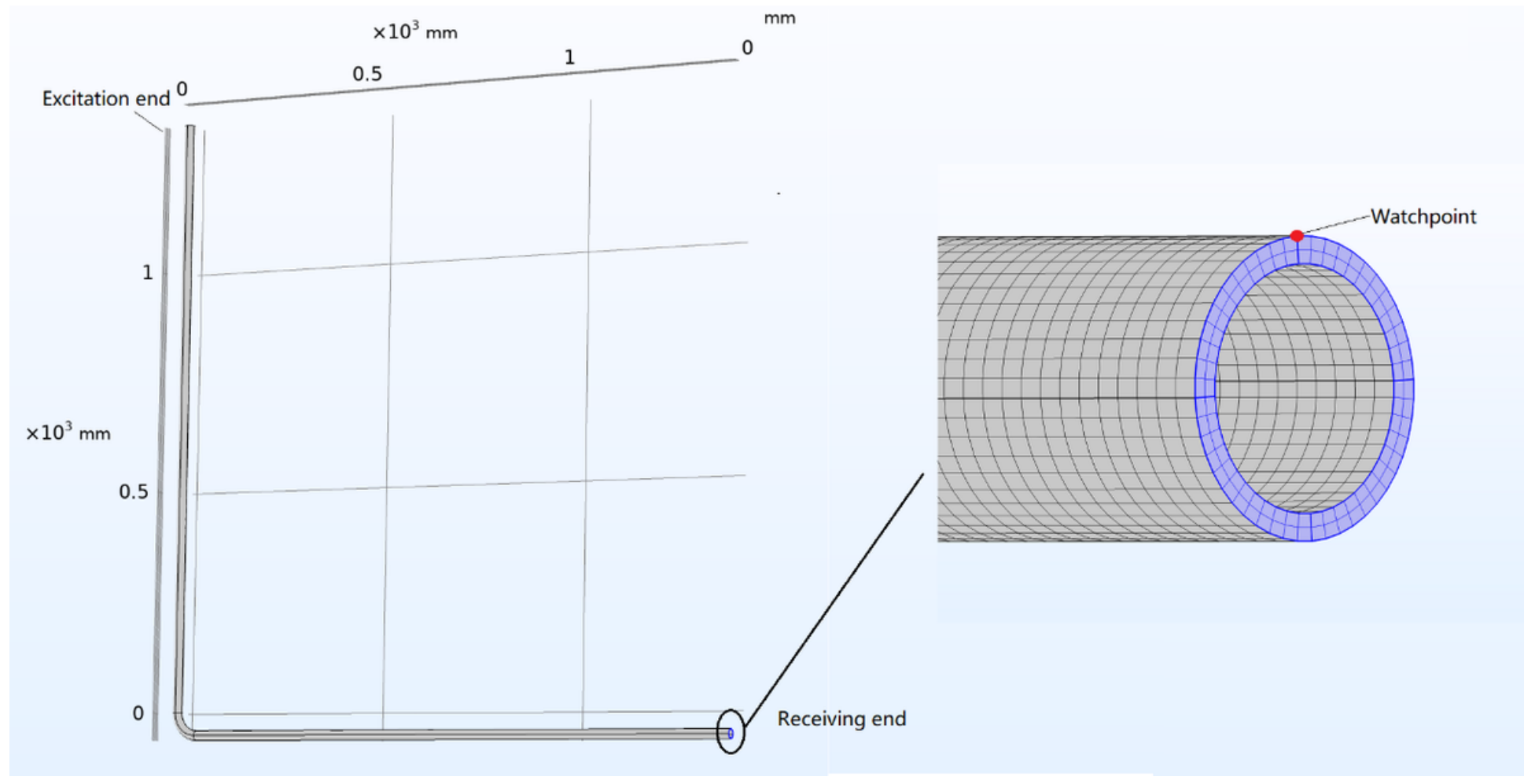

\section{Figure 6}

Finite-element modeling of test pipe.

(a) Axial displacement of the watchpoint

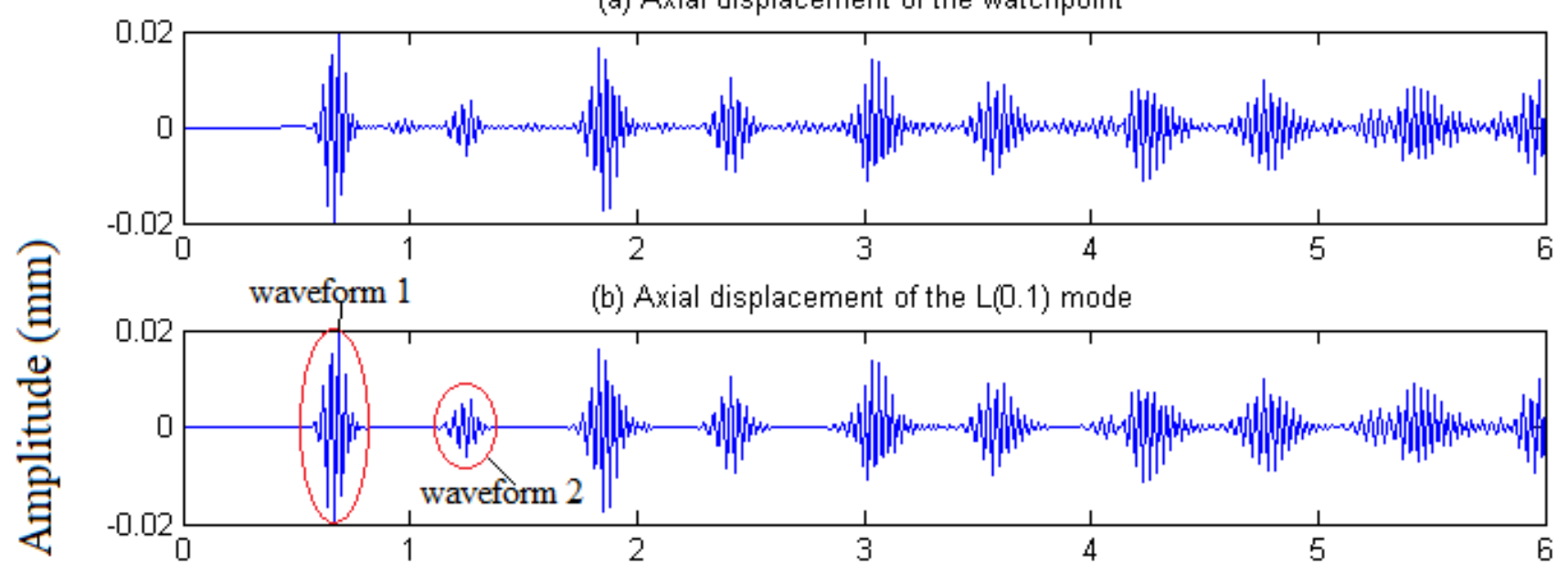

(c) Axial displacement of the $F(1,1)$ mode

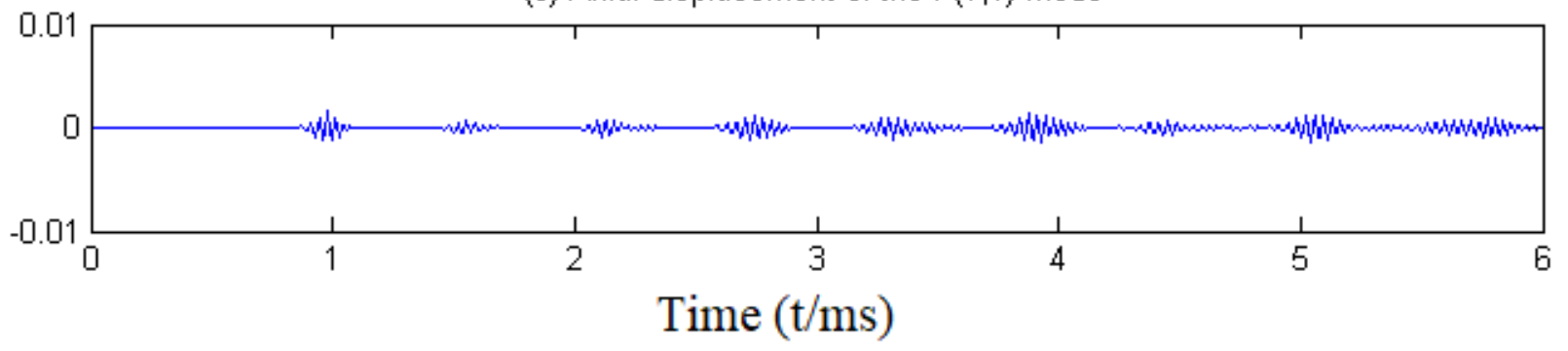




\section{Figure 7}

Time traces of axial displacement recorded at watchpoint.

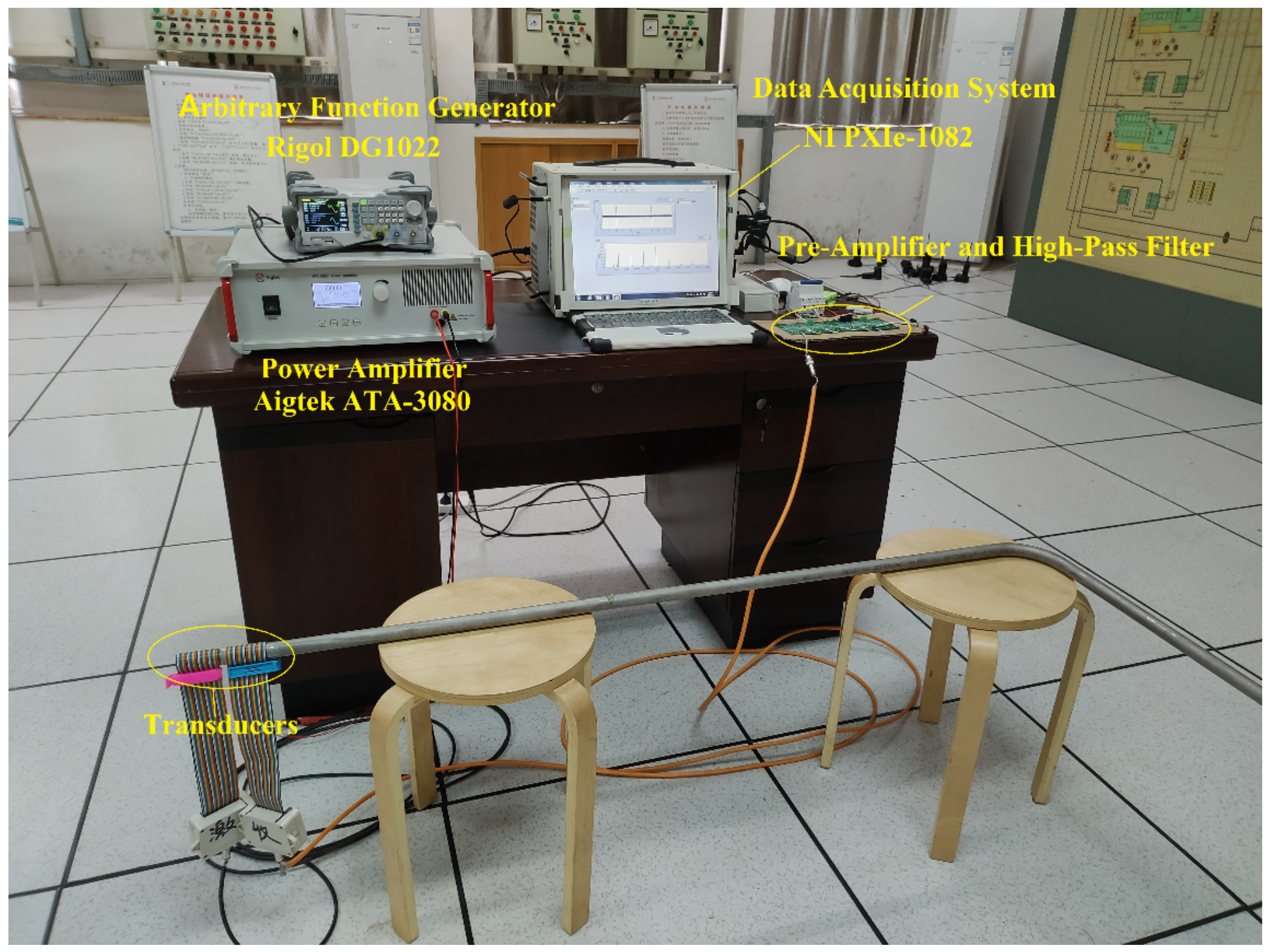

Figure 8

Experimental rig. 


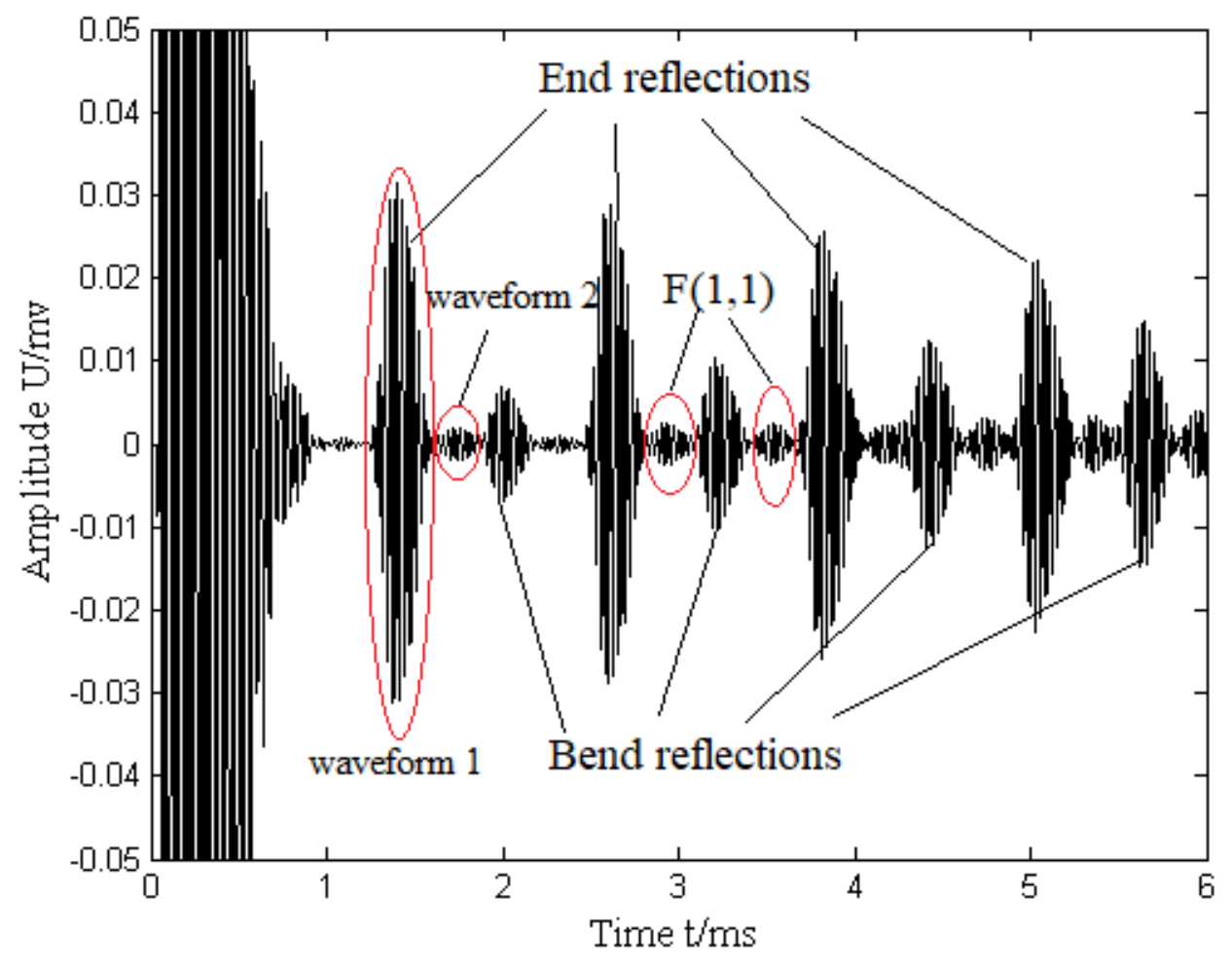

Figure 9

Guided-wave inspection of pipe with bend. 